\title{
Deep phenotyping of human induced pluripotent stem cell-derived atrial and ventricular cardiomyocytes
}

\author{
Lukas Cyganek, ${ }^{1,2}$ Malte Tiburcy, ${ }^{2,3}$ Karolina Sekeres, ${ }^{4}$ Kathleen Gerstenberg, ${ }^{1}$ \\ Hanibal Bohnenberger, ${ }^{5}$ Christof Lenz, ${ }^{6,7}$ Sarah Henze, ${ }^{1}$ Michael Stauske, ${ }^{1,2}$ Gabriela Salinas, ${ }^{8}$ \\ Wolfram-Hubertus Zimmermann, ${ }^{2,3}$ Gerd Hasenfuss, ${ }^{1,2}$ and Kaomei Guan 1,2,4 \\ ${ }^{1}$ Clinic for Cardiology and Pneumology, University Medical Center Göttingen (UMG), Göttingen, Germany. ${ }^{2} \mathrm{DZHK}$ (German \\ Center for Cardiovascular Research), partner site Göttingen, Germany. Institute of Pharmacology and Toxicology, UMG \\ Göttingen, Germany. ${ }^{4}$ Institute of Pharmacology and Toxicology, Technische Universität Dresden, Dresden, Germany. \\ Institute of Pathology, UMG, Göttingen, Germany. IInstitute for Clinical Chemistry, UMG, Göttingen, Germany. \\ ${ }^{7}$ Bioanalytical Mass Spectrometry, Max Planck Institute for Biophysical Chemistry, Göttingen, Germany. ${ }^{8}$ Transcriptome \\ and Genome Analysis Laboratory Core Unit, UMG, Göttingen, Germany.
}

Generation of homogeneous populations of subtype-specific cardiomyocytes (CMs) derived from human induced pluripotent stem cells (iPSCs) and their comprehensive phenotyping is crucial for a better understanding of the subtype-related disease mechanisms and as tools for the development of chamber-specific drugs. The goals of this study were to apply a simple and efficient method for differentiation of iPSCs into defined functional CM subtypes in feeder-free conditions and to obtain a comprehensive understanding of the molecular, cell biological, and functional properties of atrial and ventricular IPSC-CMs on both the single-cell and engineered heart muscle (EHM) level. By a stage-specific activation of retinoic acid signaling in monolayer-based and well-defined culture, we showed that cardiac progenitors can be directed towards a highly homogeneous population of atrial CMs. By combining the transcriptome and proteome profiling of the iPSC-CM subtypes with functional characterizations via optical action potential and calcium imaging, and with contractile analyses in EHM, we demonstrated that atrial and ventricular iPSC-CMs and -EHM highly correspond to the atrial and ventricular heart muscle, respectively. This study provides a comprehensive understanding of the molecular and functional identities characteristic of atrial and ventricular iPSC-CMs and -EHM and supports their suitability in disease modeling and chamber-specific drug screening.

Conflict of interest: WHZ is a cofounder and, together with MT, scientific advisor of Myriamed and Repairon.

Submitted: January 22, 2018

Accepted: May 17, 2018

Published: June 21, 2018

\section{Reference information:}

JCI Insight. 2018;3(12):e99941. https://doi.org/10.1172/jci.

insight.99941.

\section{Introduction}

Human induced pluripotent stem cell-derived (iPSC-derived) cardiomyocytes (CMs) as well as engineered heart muscle (EHM) offer great potential for the study of cardiac development and disease modeling (1) and for drug discovery and cardiotoxicity screenings in a human physiologically relevant model system (2). Several protocols are available to efficiently differentiate human iPSCs into CMs, which possess structural and functional properties similar to human embryonic CMs $(3,4)$. The generation of homogeneous populations of subtype-specific iPSC-CMs and their comprehensive phenotypic comparison are crucial for a better understanding of the predominantly cardiac subtype-restricted disease mechanisms and the development of chamber-specific drugs.

The human heart is composed of a highly diversified set of both muscle and non-muscle cell types including chamber-specific $\mathrm{CM}$ subtypes differing in both function and localization. Various signaling pathways including WNT, TGF- $\beta$, and BMP have been identified as being crucial in the guiding of stem cells into the cardiac lineages $(5,6)$. Retinoic acid (RA), the biologically active derivative of vitamin $\mathrm{A}$, has been implicated in various steps of cardiovascular development and atrial chamber formation, both in vivo and in vitro (7-10). Recently, several protocols have been published to efficiently and cost-effectively differentiate human embryonic stem cells (ESCs) or iPSCs towards the ventricular lineage, showing that over $90 \%$ of differentiated cells are positive for cardiac troponin T (cTNT) (11-13). However, protocols for atrial CM 
differentiation appear less efficient than those for ventricular CMs, giving approximately $50 \%-65 \% \mathrm{cTNT}^{+}$ CMs $(9,14)$, or apply a cocktail of cytokines and growth factors using the embryoid body method (10, 14, 15), which are cost-intensive and laborious. The phenotyping of the generated atrial and ventricular iPSCCMs was mainly based on electrophysiological measurements and gene expression profiles of subtypespecific markers (16-18). A comprehensive characterization and direct comparison of iPSC-CM subtype function and proteome assembly in atrial and ventricular CMs and EHM has, to our knowledge, not been performed so far and will be key for their further exploitation in disease modeling and drug development.

In this study, we applied a simplified, reproducible, and robust method for the directed in vitro differentiation of human iPSCs into defined CM subtypes under feeder-free culture conditions. We investigated the molecular, cell biological, and functional characteristics of the atrial and ventricular iPSC-CMs on both the single-cell and tissue level. By combining the transcriptome and proteome profiling of the iPSC-CM subtypes with a functional characterization via optical action potential (AP) and calcium imaging, as well as with a contractile analysis in EHM, we demonstrate that atrial and ventricular iPSC-CMs and -EHM highly correspond to the atrial and ventricular heart muscle, respectively. Our data further support the suitability of in vitro-generated human iPSC-CM subtypes for their application in disease modeling and drug screening.

\section{Results}

Efficient generation of atrial and ventricular iPSC-CMs. Three iPSC lines (iWT.D2.1, iBM76.3, and isWT1.Bld2) derived from 3 individuals and cultured in the feeder-free condition were characterized for their pluripotency prior to differentiation experiments (Supplemental Figure 1; supplemental material available online with this article; https://doi.org/10.1172/jci.insight.99941DS1). To effectively differentiate iPSCs into atrial and ventricular iPSC-CMs, we modified previously published protocols to allow for controlled, monolayer-based directed differentiation via modulation of WNT signaling, as previously described $(11,13)$, and applied RA to induce atrial iPSC-CMs (Figure 1A) $(9,10)$. First, we systematically evaluated the ideal time window and concentration of RA for driving atrial differentiation. Our data suggested that treatment with $1 \mu \mathrm{M}$ RA at days 3-6 resulted in enhanced expression of atrium-specific genes such as MYL7, GJA5, and NR2F2, but reduced expression of ventricle-specific genes MYL2, GJA1, and IRX4 in CM cultures at days 30 and 60, when compared with the control cultures without RA treatment (Supplemental Figure 2).

To evaluate the role of regulatory elements of WNT and RA signaling during initial stages of differentiation, we analyzed expression of WNT-associated genes $W N T 3 A, W N T 8 A$, and $L E F 1$ as well as RA signaling-related genes HOXA1 and RARB (Figure 1, B and C). Our data showed that WNT3A and WNT8A were only transiently induced at day 2 and were downregulated from day 4 during differentiation, independent of RA treatment. LEF1 was induced from day 2 and showed no significant difference between control and RA-treated cells until day 14. The RA downstream target HOXA1 was also transiently induced at day 2 in the control culture but showed persistent high expression in RA-treated cells until day 6 and then was downregulated (Figure 1, B and C). Moreover, we observed higher expression of RA receptor $R A R B$ from day 4 on in RA-treated cultures compared with control cells (Figure 1, B and C).

To assess the CM differentiation efficiency, the percentage of cTNT-expressing cells was measured after 20-day differentiation without metabolic selection with lactate. Whereas control cultures showed $89.0 \% \pm$ $2.9 \% \mathrm{cTNT}^{+} \mathrm{CMs}$, treatment with $1 \mu \mathrm{M} \mathrm{RA}$ at days $3-6$ generated $87.1 \% \pm 2.5 \% \mathrm{cTNT}^{+} \mathrm{CMs}$ without substantial effects on the total cell number (Figure 1D). Metabolic selection by replacing glucose with lactate from days 15-20 during differentiation only very slightly enhanced the purity of CMs to $90.5 \% \pm 1.3 \% \mathrm{cTNT}^{+}$ cells in RA-treated cultures, and to $90.4 \% \pm 2.1 \% \mathrm{cTNT}^{+}$cells in non-RA-treated cultures (Figure 1D).

Immunocytochemical staining of cardiac subtype-specific proteins showed that $77.8 \% \pm 1.7 \%$ of nonRA-treated (control) cells were stained positive for the ventricular isoform of myosin light chain 2 and negative for the atrial isoform of myosin light chain $2\left(\mathrm{MLC}^{+} \mathrm{V}^{+} / \mathrm{MLC}^{2} \mathrm{~A}^{-}\right)$. A substantially smaller fraction of cells, $10.9 \% \pm 1.6 \%$, displayed an immature ventricular phenotype positive for both MLC2V and MLC2A $\left(\mathrm{MLC}^{2} \mathrm{~V}^{+} / \mathrm{MLC}^{2} \mathrm{~A}^{+}\right)$, whereas $11.3 \% \pm 1.4 \%$ of iPSC-CMs from the control group were only positive for MLC2A (MLC2V $\mathrm{V}^{-} / \mathrm{MLC}^{2} \mathrm{~A}^{+}$) at around day 100 (Figure 2, A and C). In contrast, iPSC-CMs of the RA-treated group showed opposite outcomes with $4.5 \% \pm 0.8 \% \mathrm{MLC}^{2} \mathrm{~V}^{+} / \mathrm{MLC}^{2} \mathrm{~A}^{-}, 0.8 \% \pm 0.4 \%$

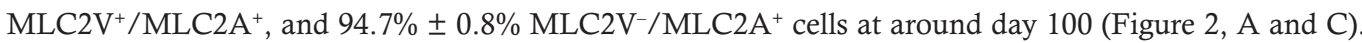
A previous study showed that MLC2V is ventricularly restricted and that MLC2A is highly expressed in the human atrium, but at low levels in the human ventricle (19). Both iPSC-CM subtypes displayed wellorganized striated patterns visualized by $\alpha$-actinin and F-actin staining and pronounced expression of the 
A

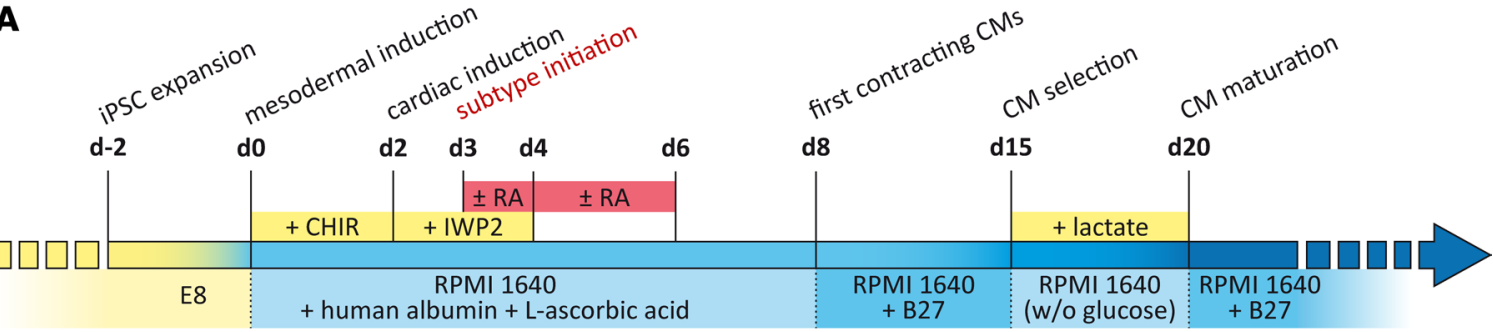

B

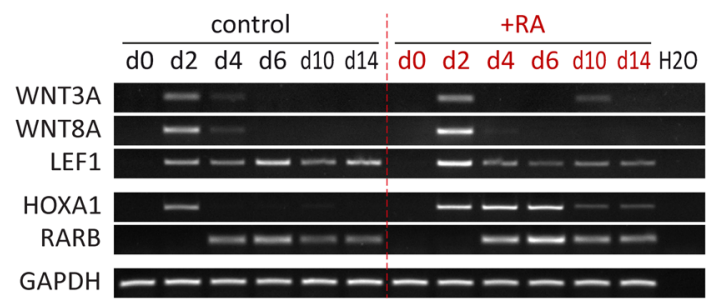

D
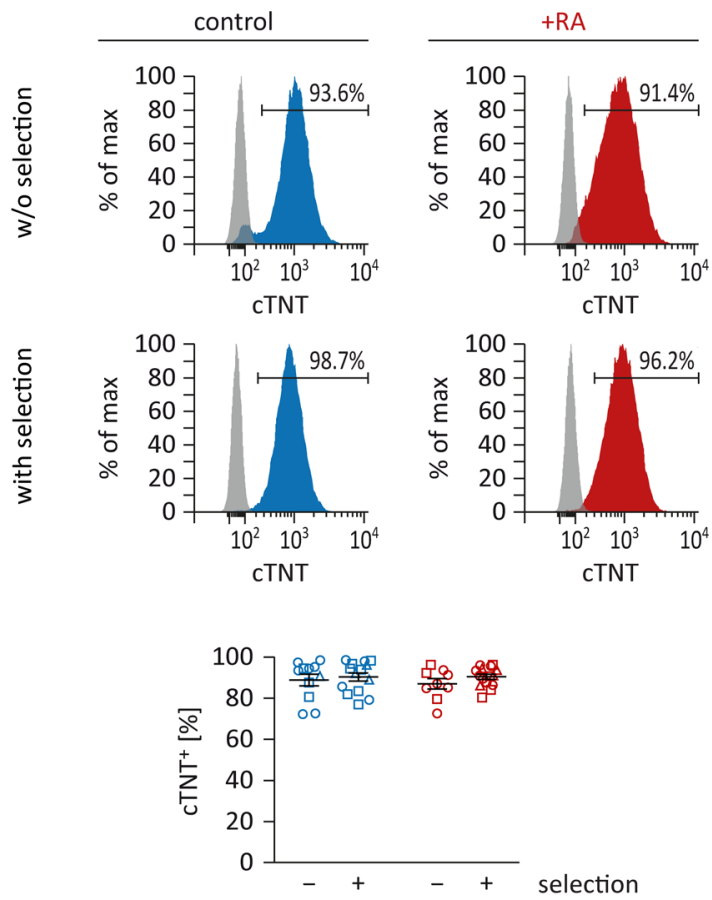

control
$+\mathrm{RA}$

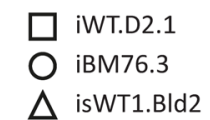

C

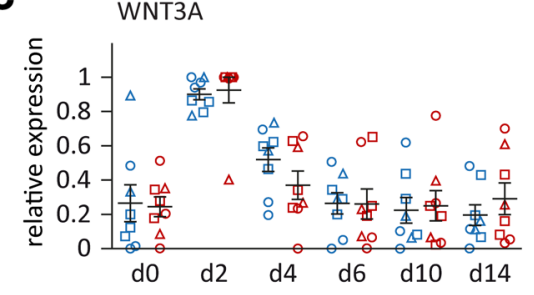

WNT8A

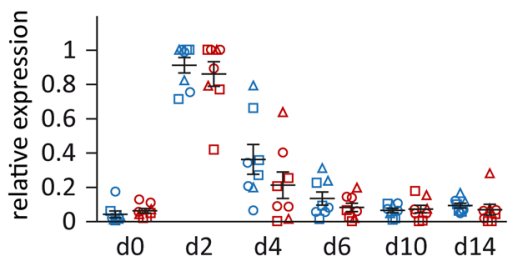

LEF1

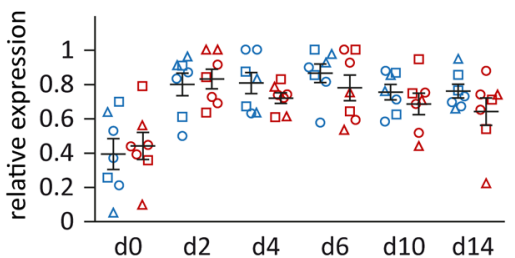

HOXA1

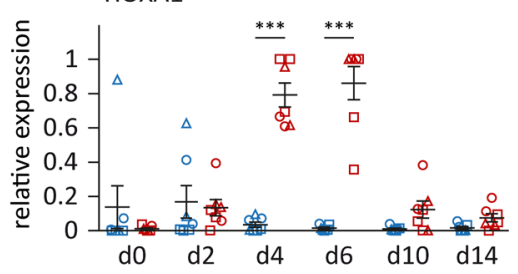

RARB

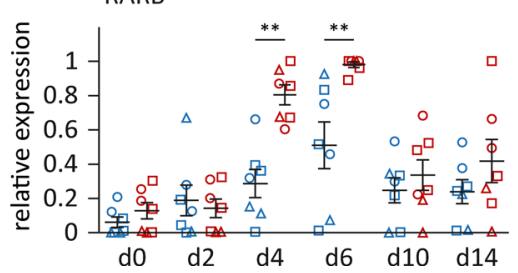

Figure 1. Directed differentiation of iPSCs into atrial or ventricular cardiomyocytes (CMs). (A) Schematic of the defined differentiation protocols. Small molecules CHIR and IWP2, which modulate canonical WNT signaling, were applied for the induction of iPSCs into CMs. Retinoic acid (RA) was used to induce the atrial subtype specification. Metabolic selection with lactate was applied to achieve a higher purity of iPSC-CMs. (B and C) Expression of genes involved in WNT and RA signaling was assessed by reverse transcriptase PCR analysis in control and RA-treated cells. Shown are representative images (B, iPSC line iBM76.3) and semiquantitative analysis of gene expression (C, $n=2-3$ independent differentiation experiments performed for each iPSC line; 3 iPSC lines were used). Results were quantified according to intensity and normalized to GAPDH expression. (D) Flow cytometry analysis of iPSC-CMs with or without lactate selection for cardiac troponin T (cTNT). Top: Representative measurements (iPSC line iBM76.3); gray peaks represent the isotype control. Bottom: Quantitative analysis ( $n=11-14$ control and $n=9-15$ RA-treated independent differentiation experiments from 3 iPSC lines). Data are presented as mean \pm SEM. ${ }^{* *} P<0.01,{ }^{* *} P<0.001$ by nonparametric Mann-Whitney $U$ test. 
A
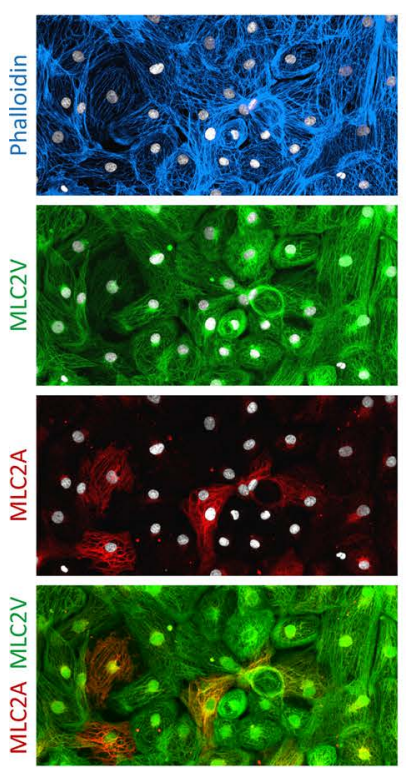

$+\mathrm{RA}$

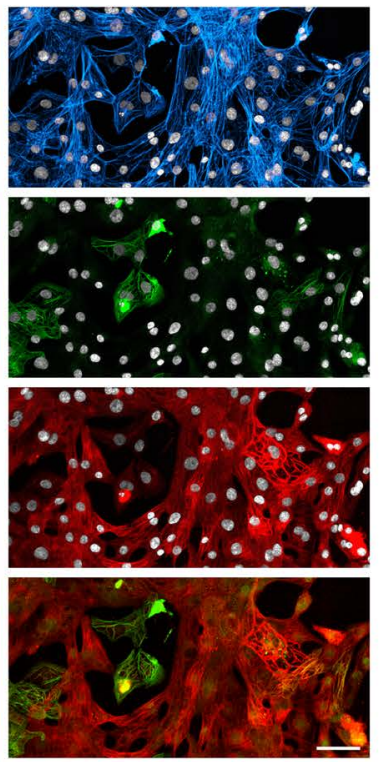

B

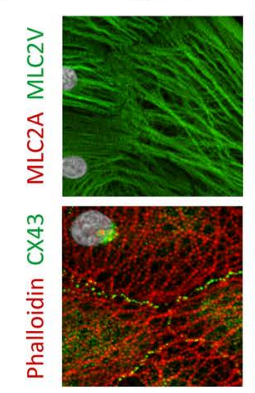

$+\mathrm{RA}$

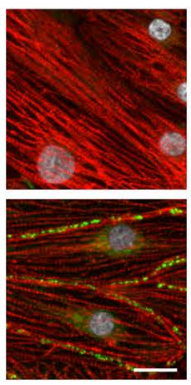

control

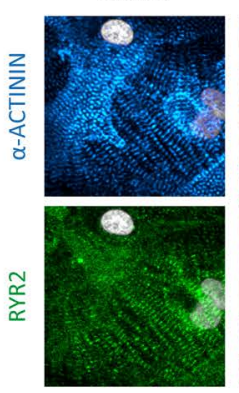

$+\mathrm{RA}$

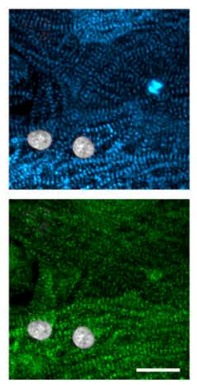

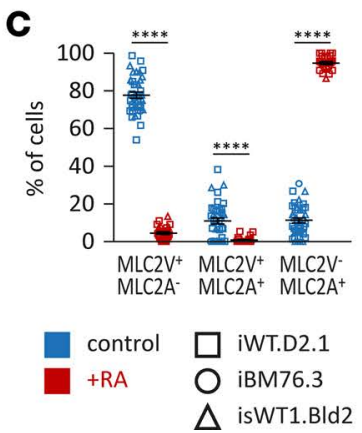

D

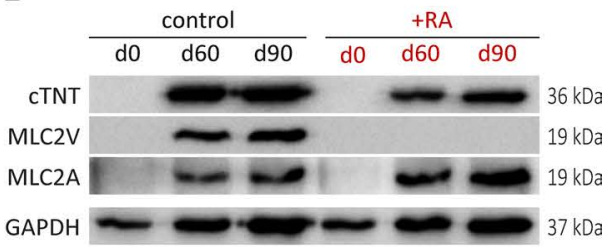

Figure 2. Structural characterization of iPSC-derived atrial or ventricular cardiomyocytes. (A) Immunofluorescence staining data of retinoic acid-treated (RA-treated) and untreated iPSC-derived cardiomyocyte (iPSC-CMs) cultures for F-actin (phalloidin), MLC2V, and MLC2A (iPSC line iWT.D2.1, day 86); nuclei were stained with DAPI (white). Scale bar: $50 \mu \mathrm{m}$. (B) Structural characterization via immunofluorescence staining for F-actin, $\alpha$-actinin, MLC2V, MLC2A, gap junction protein CX43, and sarcoplasmic reticulum calcium channel RYR2 (iPSC lines iWT.D2.1 and isWT1.BId2); nuclei were stained with DAPI (white). Scale bar: $20 \mu \mathrm{m}$. (C) Quantification of MLC2V ${ }^{+}$and MLC2A+ cells in immunofluorescently stained cultures ( $n=15$ control and $n=11$ RA-treated independent differentiation experiments from 3 iPSC lines, days $80-120$ ). Data are presented as mean \pm SEM. ${ }^{* * * *} P<0.0001$ by nonparametric Mann-Whitney $U$ test. (D) Western blot analysis for general cardiac marker cardiac troponin T (cTNT) and subtype markers MLC2V and MLC2A at days 60 and 90 (iPSC line iWT.D2.1).

gap junction protein connexin 43 (CX43) and sarcoplasmic reticulum calcium channel ryanodine receptor 2 (RYR2), indicating expression of proteins important for intact myofibril and sarcoplasmic reticulum function in both subtypes (Figure 2B). These observations were confirmed by Western blot analysis. Whereas iPSC-CMs generated under the control condition showed robust expression of ventricle-specific MLC2V and lower levels of MLC2A, iPSC-CMs obtained from the RA-treated cultures revealed high expression of MLC2A, but no expression of MLC2V (Figure 2D). In summary, these data demonstrate that RA treatment effectively induces the generation of atrial iPSC-CMs (iPSC-aCMs) when ventricular iPSC-CMs (iPSC-vCMs) are mainly generated under the control condition without RA treatment.

Transcriptome and proteome analysis of atrial and ventricular iPSC-CMs. In order to deeply characterize the generated iPSC-aCMs and -vCMs, we performed transcriptome and proteome analyses. Comparison of total coding and noncoding RNA levels in iPSC-aCMs with those in iPSC-vCMs at day 90 revealed 1,902 transcripts with significant and more than 2-fold changes in expression levels (1,037 upregulated and 865 downregulated) (Supplemental Table 1). Even though they were derived from different iPSC lines and different differentiation experiments, the principal component analysis (PCA) and the cluster dendrogram of the global gene expression data illustrated a clear separation of the iPSC-aCM samples from the iPSC-vCM samples independent of the different origins of the iPSC lines used (Figure 3, A and B, and Supplemental Figure 3A). These data supported the notion that the differential expression resulted from the 2 efficient and reproducible differentiation protocols and not from interindividual genotypic differences. The most regulated genes specific for ventricular or atrial CMs are shown in a heatmap (Figure $3 \mathrm{C}$ ). Genes associated with human atrial CMs (HEY1, NR2F1, NR2F2, TBX5, ATP2A2, and MYH6) showed significantly higher expression in iPSC-aCMs compared with iPSC-vCMs (Figure 3C). Strikingly, we identified genes encoding potassium channels $\mathrm{K}_{\mathrm{v}} 1.5(K C N A 5), \mathrm{K}_{\mathrm{ir}} 3.1(K C N J 3)$, and $\mathrm{K}_{2 \mathrm{p}} 3.1(K C N K 3)$ as displaying significantly higher expression in iPSC-aCMs compared with iPSC-vCMs. These channels contribute to the APs in human atria by being responsible for the ultrarapid delayed rectifier $\mathrm{K}^{+}$current $\left(I_{K u r}\right)$, the acetylcholine-activated $\mathrm{K}^{+}$ current $\left(I_{K A C h}\right)$, and the 2-pore domain channel-mediated $\mathrm{K}^{+}$current $\left(I_{K 2 P}\right)$, respectively $(20-22)$. In contrast, 

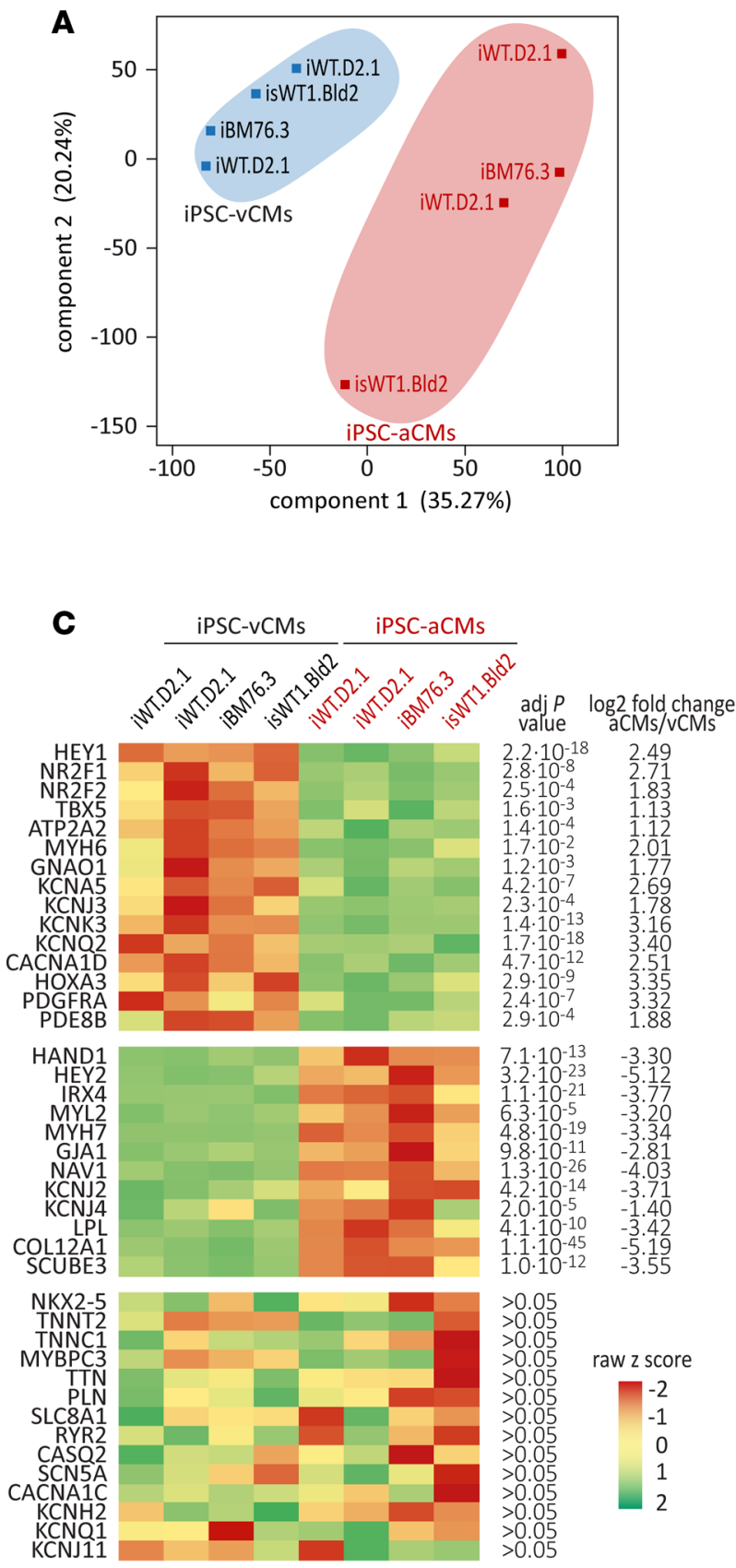

B

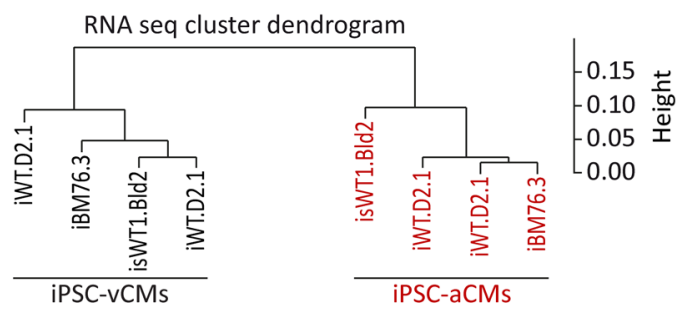

D
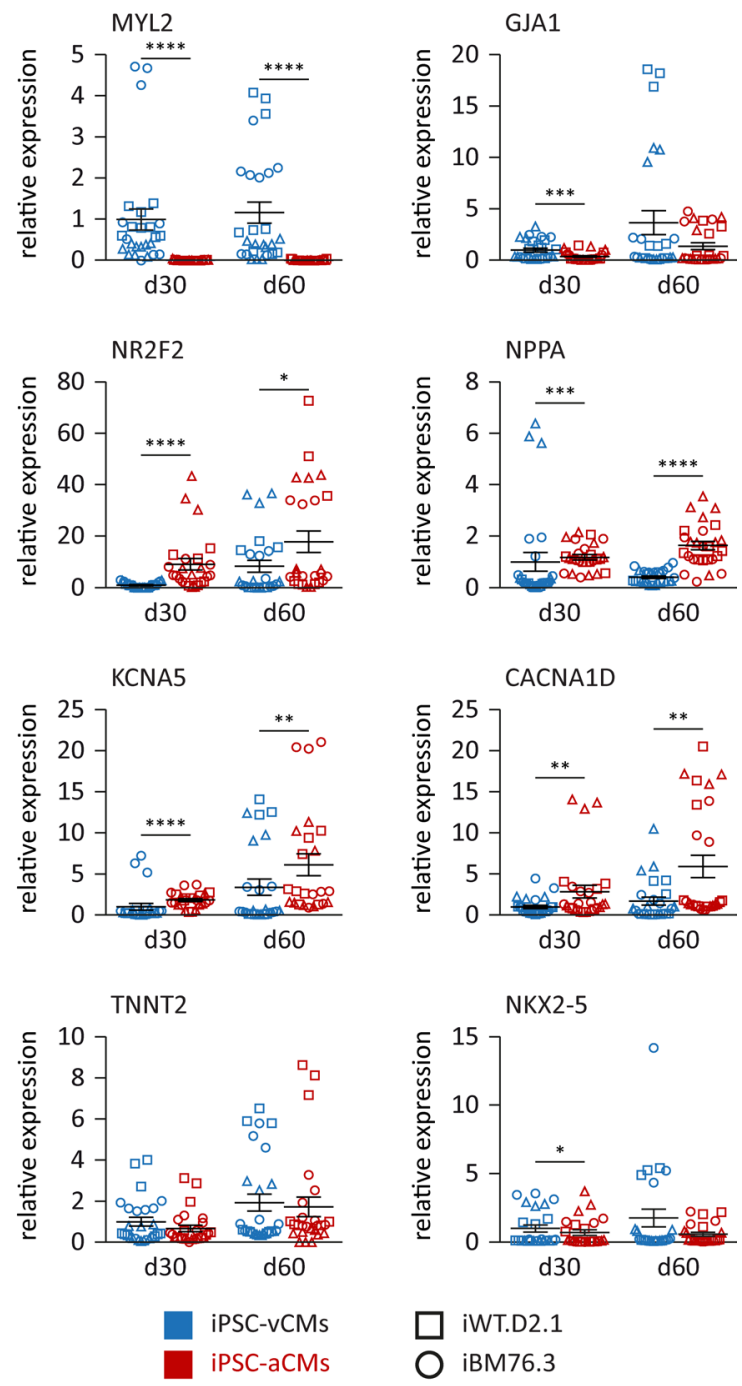

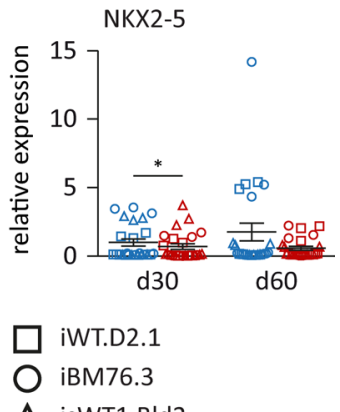

Figure 3. Transcriptome analysis of iPSC-derived atrial or ventricular cardiomyocytes. (A and B) Principal component analysis of component 1 versus component 2 (A) and cluster dendrogram (B) of the global gene expression data of iPSC-derived of atrial (iPSC-aCMs) and ventricular cardiomyocytes (iPSC-vCMs) at day 90 illustrate separation of the samples based on different atrial and ventricular differentiation protocols $(n=4 \mathrm{iPSC}-\mathrm{vCM}$ and $n=4$ iPSC-aCM independent differentiation experiments from 3 iPSC lines). (C) Heatmap of gene expression of upregulated (upper panel) and downregulated (middle panel) genes as well as general cardiac genes (lower panel) in iPSC-aCMs compared with iPSC-vCMs. Adjusted $P$ value and log2 fold change of the 2 groups are indicated; color code according to $z$ raw score. (D) Real-time PCR of selected ventricular (MYL2 and GJA1) and atrial markers (NR2F2, NPPA, KCNA5, and CACNA1D) and general cardiac genes (TNNT2 and NKX2-5) at days 30 and 60 ( $n=3$ independent differentiation experiments with triplicates each for each iPSC line; 3 iPSC lines were used); normalized to GAPDH expression and to controls at day 30 . Data are presented as mean \pm SEM. ${ }^{*} P<$ $0.05,{ }^{* *} P<0.01,{ }^{* *} P<0.001,{ }^{* * *} P<0.0001$ by nonparametric Mann-Whitney $U$ test. 
A

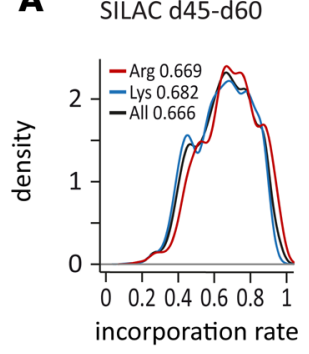

SILAC d30-d60

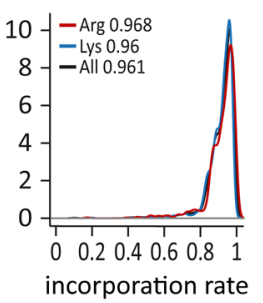

SILAC d15-d60

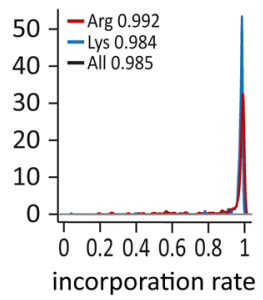

B

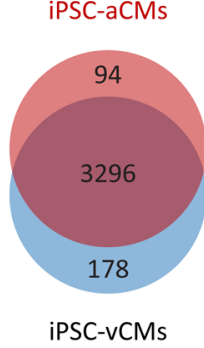

C

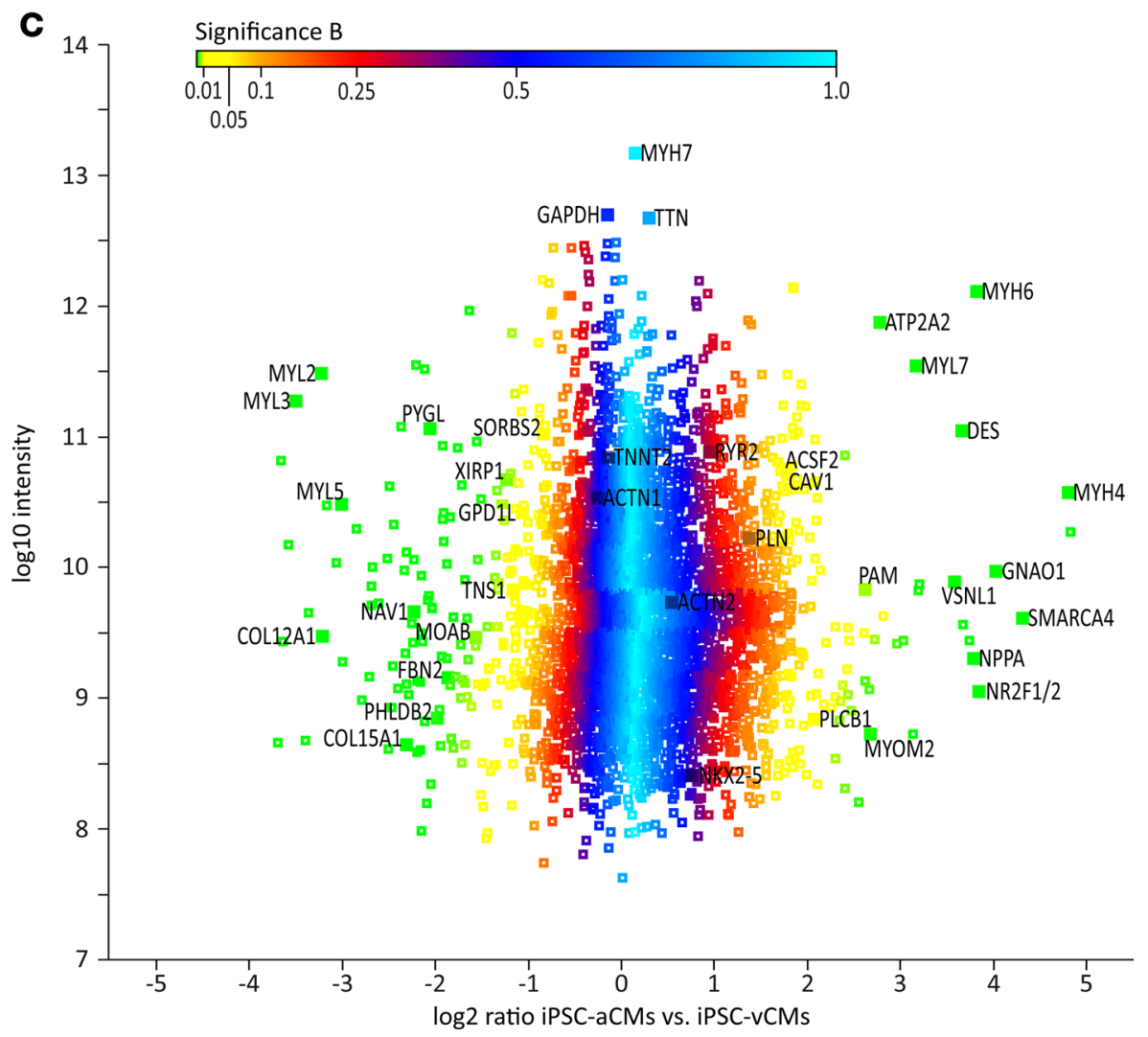

Figure 4. Stable-isotope labeling by amino acids in cell culture-based (SILAC-based) proteomics of iPSC-derived atrial or ventricular cardiomyocytes via quantitative mass spectrometry. (A) Analysis of labeling efficiency of iPSC-derived atrial and ventricular cardiomyocytes (iPSC-aCMs and iPSC-vCMs) after exposure in SILAC medium for 15 days (d45-d60), 30 days (d30-d60), and 45 days (d15-d60) prior to analysis (iPSC line isWT1.Bld2); incorporation rates of heavy-labeled lysine, arginine, and mean are indicated. (B) A total of 3,568 proteins were identified in SILAC-based proteomic analysis of iPSC-CMs after exposure in SILAC medium from days 15 to 68 , with 94 upregulated and 178 downregulated proteins in iPSC-aCMs compared with iPSC-vCMs ( $n=$ 4 iPSC-vCM and $n=4$ iPSC-aCM independent differentiation experiments from iPSC line isWT1.BId2, day 68). (C) Distribution of log2 protein ratios between iPSC-aCMs and iPSC$\mathrm{vCMs}$ versus $\log 10$ protein intensity. Selected markers are indicated; color code according to Significance B.

genes associated with the ventricular CM subtype including developmental markers (HAND1, HEY2, and IRX4) and functional genes (MYL2, MYH7, GJA1, and KCNJ2) showed significantly higher expression in iPSC-vCMs compared with iPSC-aCMs (Figure 3C). General cardiac-specific genes like NKX2-5, TNNT2, $P L N$, or $C A S Q 2$ were detected with no significant differences between iPSC-aCMs and iPSC-vCMs, indicating a cardiac phenotype and comparable proportions of functional CMs in the samples (Figure 3C). RNA sequencing data of selected general and subtype-specific markers were verified by real-time PCR (Figure 3D). Gene ontology (GO) enrichment analysis of the top 200 differentially expressed genes between iPSC-aCMs and iPSC-vCMs showed that these genes were mainly involved in ion channel functions, cellcell signaling, calcium handling, contraction, morphogenesis, and development of CMs (Supplemental Figure 4). Furthermore, we performed comparison analysis of our RNA sequencing data with the published transcriptomic data sets from human ESC-derived atrial and ventricular CMs at day 31 after differentiation (14), 15-week-old human fetal atrial and ventricular tissues (14), and human adult atrial and ventricular tissues (23), respectively. We found a strong overlap and correlation of subtype-specific candidate genes in our study with the published data (Supplemental Figure 3, B-D, and Supplemental Tables 2-4).

To quantitatively compare the proteome profile of iPSC-aCMs and -vCMs, we applied stable-isotope labeling by amino acids in cell culture (SILAC), which is a powerful approach to investigate the proteome with high resolution by incorporating stable-isotope-containing amino acids in newly synthesized proteins, allowing not only identification but also precise quantification of the proteins (24). In contrast to chemical 
or label-free methods, the SILAC-based method also allows the recording of low-abundance proteins with high reproducibility and accuracy $(24,25)$. Efficient SILAC labeling requires high protein turnover and is thought to be most effective in proliferating cells. Since iPSC-CMs only poorly divide once the differentiation process is completed, we evaluated the necessity of culturing iPSC-CMs in SILAC medium for 15 days (d45-d60), 30 days (d30-d60), and 45 days (d15-d60) in order to reach adequate labeling efficiency. By prolonged culture of iPSC-CMs in SILAC medium for 45 days, we obtained an incorporation rate for heavily labeled arginine and lysine of $98.5 \%$ in our samples, sufficient for proteomic analyses, suggesting a nearly complete turnover of all CM proteins within this timeframe (Figure 4A). Analysis of equal portions of SILAC-labeled (d15-d68) iPSC-aCMs and unlabeled iPSC-vCMs, and vice versa, allowed calculation of abundance ratios based on their mass differences and subsequently displayed the protein expression differences of the $2 \mathrm{CM}$ subtypes. We identified 3,568 proteins present in our samples, 94 of which showed significantly higher expression and 178 of which showed significantly lower expression in iPSC-aCMs compared with iPSC-vCMs at day 68 (Figure 4, B and C, and Supplemental Table 5).

To assess the correlation of our transcriptome and proteome data with respect to the atrial and ventricular markers, we performed comparison analysis of 2 data sets. We found a list of 67 markers with significantly lower expression and a list of 24 candidates with significantly higher expression in iPSC-aCMs compared with iPSC-vCMs at both the RNA and protein level (Supplemental Figure 3D and Supplemental Table 6). Furthermore, we performed the comparison analysis of our SILAC data with the published proteome data sets obtained from human fetal (26) and adult atrial and ventricular tissues (27) (Supplemental Table 6). Consistent with the previous proteome studies using human fetal (26) and adult atrial and ventricular tissues (27), atrium-enriched proteins including MLC2A (MYL7), natriuretic peptide A (NPPA), COUPTFI/II (NR2F1/2), sarcoplasmic/endoplasmic reticulum calcium ATPase 2 (ATP2A2), myosin heavy chain 4 (MYH4), $\alpha$-MHC (MYH6), guanine nucleotide-binding protein G(o) subunit $\alpha$ (GNAO1), peptidyl-glycine $\alpha$-amidating monooxygenase (PAM), 1-phosphatidylinositol 4,5-bisphosphate phosphodiesterase $\beta-1$ (PLCB1), and acyl-CoA synthetase family member 2 (ACSF2) showed significantly higher expression in iPSC-aCMs, whereas ventricle-enriched proteins including MLC2V (MYL2), MLC1V (MYL3), MLC5 (MYL5), pleckstrin homology-like domain family B member 2 (PHLDB2), sorbin and SH3 domain-containing protein 2 (SORBS2), glycogen phosphorylase (PYGL), and neuron navigator 1 (NAV1) showed significantly higher expression in iPSC-vCMs (Figure 4C and Supplemental Table 6). General cardiac markers like NKX2-5, titin (TTN), cTNT (TNNT2), phospholamban (PLN), RYR2, and $\alpha$-actinin (ACTN2) were found at equal expression levels between the 2 groups. Interestingly, some genes that are differentially expressed in iPSC-aCMs and -vCMs, as well as in fetal atrial and ventricular tissues (26), such as caveolin-1 (CAV1), tensin 1 (TNS1), Xin actin-binding repeat-containing protein 1 (XIRP1), and glycerol-3-phosphate dehydrogenase 1-like protein (GPD1L), are not found to be differentially expressed in human adult atrial and ventricular tissues (27) (Supplemental Table 6), suggesting that these genes might be important during early chamber-specific development.

Functional analysis of atrial and ventricular iPSC-CMs. In the human heart, atrial and ventricular CMs display important differences in functional and electrophysiological properties, which play a fundamental role in excitation-contraction coupling in both CM subtypes (28). In order to allow cell-type characterization at a higher throughput, we applied simultaneous fluorescence-based measurements of APs and calcium transients (CaTs) in iPSC-CMs from days 94-141 using confocal line scan imaging, with cells that were colabeled with the voltage-sensitive dye di-8-ANEPPS and calcium indicator Rhod-2 AM. The iPSC-vCMs stimulated at $0.5 \mathrm{~Hz}$ displayed typical ventricle-like AP morphology with a plateau phase, whereas iPSC-aCMs stimulated at $0.5 \mathrm{~Hz}$ exhibited atrium-like triangular APs (Figure 5, A and B). The majority of iPSC-vCMs displayed significantly longer AP duration at 50\% repolarization (APD50) compared with iPSC-aCMs (344 $\pm 7 \mathrm{~ms}$ versus $167 \pm 5 \mathrm{~ms}$ ) (Figure 5C). The APD20/APD80 ratio, a measurement of the AP plateau, was significantly higher in iPSC-vCMs compared with iPSC-aCMs $(0.31 \pm 0.01$ versus $0.12 \pm 0.01)$, indicating subtype-specific AP morphologies (Figure 5D). Similar to AP properties, differences in CaT morphologies were also observed between iPSC-aCMs and -vCMs (Figure 5, A and B). Analysis of CaTs revealed that iPSC-aCMs exhibited a significantly faster rise time compared with iPSC-vCMs (time to peak: $150 \pm 3 \mathrm{~ms}$ versus $320 \pm 6 \mathrm{~ms}$ ) (Figure 5E). The duration of CaTs at 20\% (CaTD20: $143 \pm 5 \mathrm{~ms}$ versus $300 \pm 6 \mathrm{~ms}$ ), 50\% (CaTD50: $316 \pm 8 \mathrm{~ms}$ versus $544 \pm 9 \mathrm{~ms}$ ), and 80\% decay (CaTD80: $623 \pm 14 \mathrm{~ms}$ versus $889 \pm 11$ $\mathrm{ms}$ ) was significantly shorter in iPSC-aCMs compared with iPSC-vCMs (Figure 5F). By comparison of the spontaneous beating of the cultures, we observed pronounced differences in beating frequency between the 
A
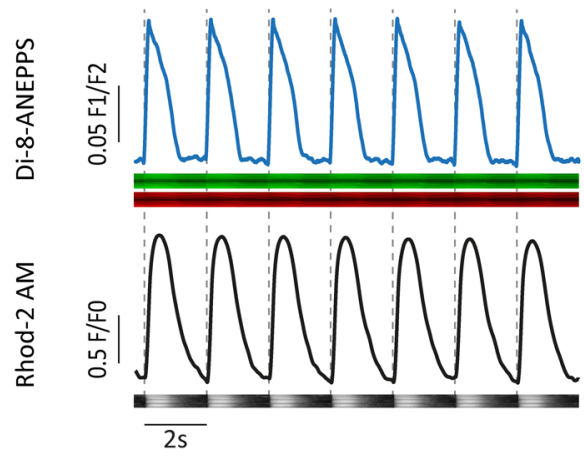

iPSC-aCMs
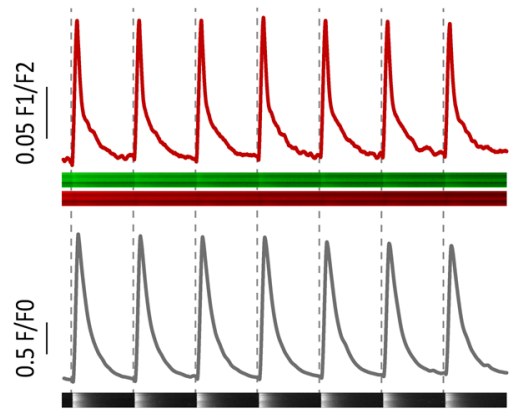

B
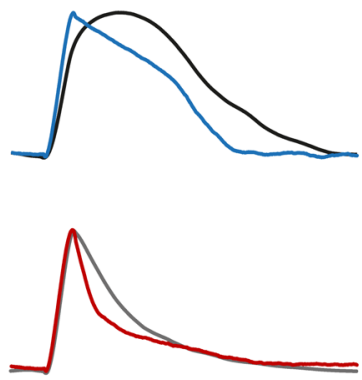

$\overline{200 \mathrm{~ms}}$
C

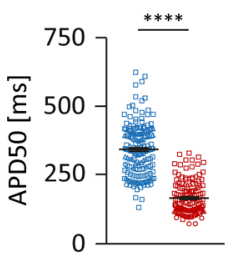

D

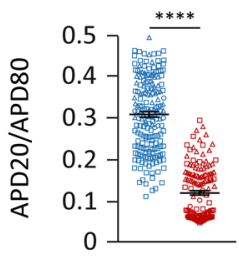

E

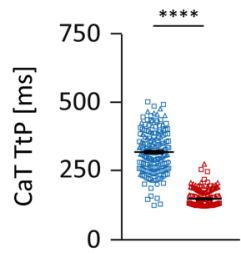

F



G

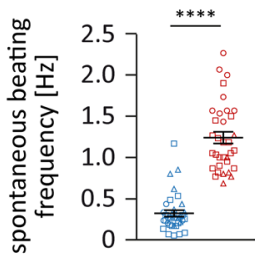

iPSC-vCMs $\square$ iWT.D2.1

iPSC-aCMs $\quad$ iBM76.3

$\triangle$ isWT1.Bld2

Figure 5. Functional analysis of iPSC-derived atrial or ventricular cardiomyocytes by optical voltage and calcium recordings. (A-F) Optical voltage (di-8-ANEPPS) and calcium (Rhod-2 AM) confocal imaging of iPSC-derived atrial and ventricular cardiomyocytes (iPSC-aCMs, iPSC-vCMs) ( $n$ = 6 iPSC-vCM and $n=4$ iPSC-aCM independent differentiation experiments from 3 iPSC lines, days 94-141); paced at 0.5 Hz. (A) Representative plots of action potentials (APs) (upper panel) and calcium transients (CaTs) (lower panel) of a single cell with original voltage-sensitive dye and calcium dye recordings below (iPSC line iWT.D2.1, days 94 and 115). (B) Overlap of normalized APs (blue, red) and CaTs (black, gray) of a single cell displays typical subtype-specific AP and CaT morphology. (C and D) AP duration at 50\% repolarization (APD50) and ratio of APD20 to APD80 ( $n=184 \mathrm{iPSC}-\mathrm{vCMs}$ and $n=148 \mathrm{iPSC}-\mathrm{aCMs}$ ). (E and F) CaT rise time (time to peak, TtP) and CaT duration at 20\% (CaTD20), 50\% (CaTD50), and 80\% (CaTD80) decay ( $n=183 \mathrm{iPSC}-\mathrm{vCMs}$ and $n=170 \mathrm{iPSC}-\mathrm{aCMs}$ ). (G) Analysis of spontaneous beating frequency of iPSC-CM cultures ( $n=16$ iPSC-vCM and $n=14$ iPSC-aCM independent differentiation experiments from 3 iPSC lines, days 60-97). Data are presented as mean \pm SEM. ${ }^{* * *} P<0.0001$ by nonparametric Mann-Whitney $U$ test $(\mathbf{C}-\mathbf{G})$.

2 subtypes, whereby iPSC-aCMs beat around 4 times faster than iPSC-vCMs $(1.21 \pm 0.11 \mathrm{~Hz}$ versus 0.33 $\pm 0.06 \mathrm{~Hz}$ ) (Figure 5G). In summary, the functional characterization of the iPSC-derived CM populations reveals that iPSC-vCMs display similar properties to CMs from the human ventricle, whereas iPSC-aCMs highly correspond to the human atrial subtype.

Functional properties of iPSC-derived atrial and ventricular EHM. Next, we investigated and compared the functional properties of EHM generated from iPSC-aCMs (iPSC-aEHM) and iPSC-vCMs (iPSC-vEHM). Both iPSC-CM populations formed EHM with similar macroscopic morphology (cross-sectional area [CSA]: $1.25 \pm 0.06 \mathrm{~mm}^{2}$ in iPSC-aEHM, $1.04 \pm 0.04 \mathrm{~mm}^{2}$ in iPSC-vEHM; Figure 6A, Supplemental Figure 5A, and Supplemental Videos 1 and 2). Microscopically, thick $\alpha$-actinin-positive CM bundles were detected in both iPSC-aEHM and -vEHM, with a clearly divergent expression pattern of subtype-specific myosin light chain isoforms (Figure 6B). Consistent with data from the monolayer cultures of iPSC-CMs, we found a higher beating frequency, a shorter contraction time, and a faster relaxation in iPSC-aEHM compared with iPSC-vEHM (Figure 6, C-E). Both iPSC-aEHM and -vEHM revealed a concentration-dependent, positive inotropic response to increasing extracellular calcium concentrations, with a half-maximal effective concentration $\left(\mathrm{EC}_{50}\right.$ ) of $0.34 \mathrm{mM}$ for iPSC-vEHM and $0.41 \mathrm{mM}$ for iPSC-aEHM (Figure 6F and Supplemental Figure 5B). The overall force of contraction (FOC) corrected by CSA was not significantly different between the 2 types of EHM (Figure 6F). The muscle content (amount of CMs) after EHM generation was slightly higher in iPSC-aEHM than in iPSC-vEHM (Supplemental Figure 5C). FOC normalized to muscle content of iPSC-aEHM was, however, lower than that of iPSC-vEHM (Supplemental Figure 5D). We also found a positive force-frequency response in iPSC-vEHM, which was absent in iPSC-aEHM (Figure 6G), consistent with earlier reports on the force-frequency behavior of adult human atrial and ventricular heart muscle $(29,30)$. 
A

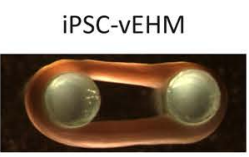

B
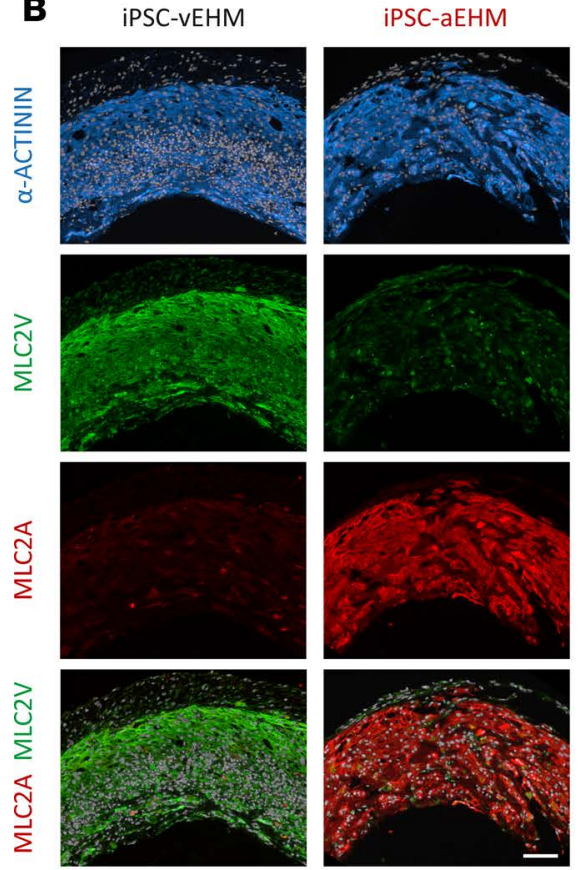

C

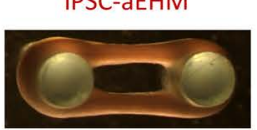

D

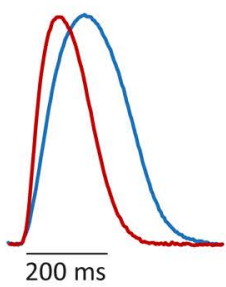

$\mathbf{F}$

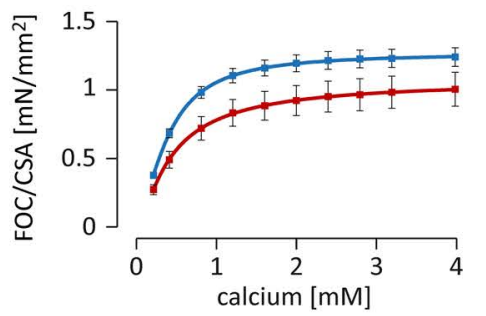

H

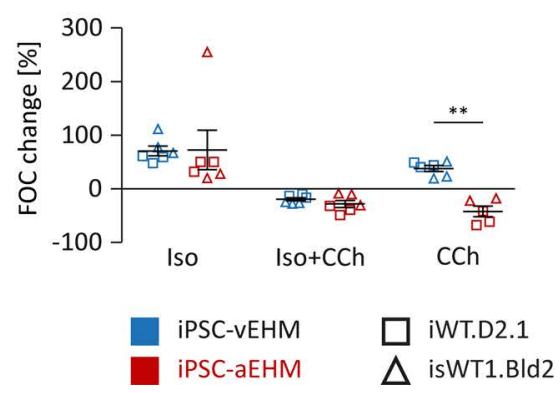

E
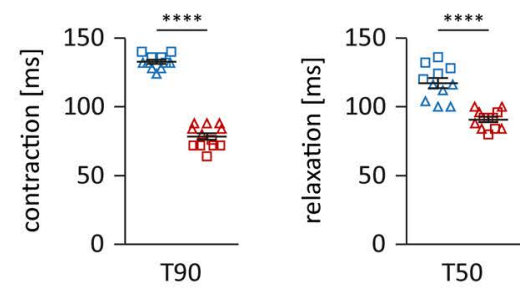

G

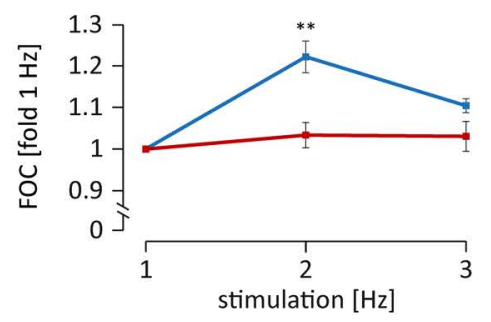

I

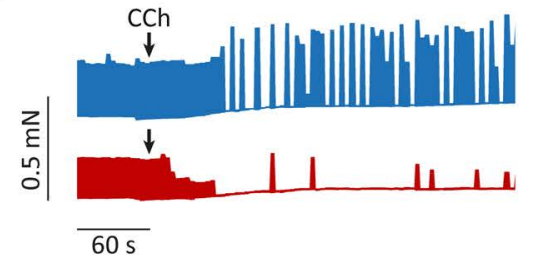

Figure 6. Morphological and functional analysis of iPSC-derived atrial or ventricular engineered heart muscle. (A) Macroscopic appearance of iPSC-derived atrial and ventricular engineered heart muscle (iPSC-aEHM and iPSC-vEHM; iPSC line iWT.D2.1). (B) Immunofluorescence staining of iPSC-EHM cross sections for cardiac marker $\alpha$-actinin, MLC2V, and MLC2A (iPSC line isWT1.BId2); nuclei were stained with DAPI (white). Scale bar: $100 \mu \mathrm{m}$. (C-G) Functional analysis of iPSC-EHM at half-maximal effective concentration $\left(E_{50}\right)$ for calcium and paced at $2 \mathrm{~Hz}$, if not indicated otherwise ( $n=11 \mathrm{iPSC}-\mathrm{vEHM}$ and $n=12 \mathrm{iPSC}-\mathrm{aEHM}$ from iPSC lines iWT.D2.1 and isWT1.BId2). (C) Analysis of spontaneous beating frequency of iPSCEHM. (D and E) Representative normalized contraction curves of iPSC-EHM and analysis of time to $90 \%$ of contraction (T90) and time to $50 \%$ of relaxation (T50). (F) Analysis of force of contraction (FOC) in relation to cross-sectional area (CSA) of iPSC-EHM in response to increasing extracellular calcium from 0.2-4 mM. (C) Analysis of force-frequency response of iPSC-EHM to increasing stimulation frequencies normalized to individual EHM at $1 \mathrm{~Hz}$. ( $\mathbf{H}$ and $\mathbf{I}$ ) Pharmacological treatment of iPSC-EHM at EC $\mathrm{F}_{50}$ calcium concentration and paced at $2 \mathrm{~Hz}$, if not indicated otherwise $(n=6$ iPSC-vEHM and $n=6$ iPSC-aEHM from iPSC lines iWT.D2.1 and isWT1.BId2). (H) Change of FOC in iPSC-EHM in response to acute stimulation with $1 \mu \mathrm{M}$ isoprenaline (Iso), $1 \mu \mathrm{M}$ Iso and $10 \mu \mathrm{M}$ carbachol (CCh), or $10 \mu \mathrm{M}$ CCh only. (I) Representative recordings from spontaneous iPSC-EHM contractions over time before and after stimulation with $10 \mu \mathrm{M}$ CCh (indicated by arrow at 60 seconds). Data are presented as mean \pm SEM. ${ }^{* *} P<0.01$, ${ }^{* *} P<0.001,{ }^{* * *} P<0.0001$ by nonparametric Mann-Whitney $U$ test $(\mathbf{C}, \mathbf{E}$, and $\mathbf{H})$ or by 2-way ANOVA with Sidak's correction (F and $\left.\mathbf{G}\right)$.

To investigate the differential pharmacological response of iPSC-aEHM and -vEHM to $\beta$-adrenergic stimulation with isoprenaline (Iso, $1 \mu \mathrm{M}$ ) and to muscarinergic stimulation with carbachol $(\mathrm{CCh}, 10 \mu \mathrm{M})$, we measured FOC and beating frequency of individual EHM at the approximate $\mathrm{EC}_{50}$ for calcium. The positive inotropic response to Iso was comparable in both $\mathrm{EHM}$ subtypes $(71 \%$ $\pm 10 \%$ increase in FOC in iPSC-vEHM vs. $73 \% \pm 37 \%$ increase in FOC in iPSC-aEHM; Figure $6 \mathrm{H}$ ). In the presence of Iso, $\mathrm{CCh}$ induced a negative inotropic effect, which was slightly more pronounced in iPSC-aEHM than in iPSC-vEHM (Figure 6H). Interestingly, when CCh was applied without prestimulation with Iso, we observed a positive inotropic response in iPSC-vEHM but a negative inotropic response in iPSC-aEHM (Figure 6H). This differential pattern has previously been identified in adult human ventricular and atrial heart muscle samples (31). Furthermore, the treatment with $10 \mu \mathrm{M} \mathrm{CCh}$ resulted in a complete block of spontaneous beating activity in iPSC-aEHM and evoked a mild negative chronotropy in iPSC-vEHM (Figure 6I). In summary, we demonstrate what we believe to be a novel strategy to engineer aEHM and vEHM with canonical properties of bona fide human atrial and ventricular myocardium, respectively. 


\section{Discussion}

In this study, we applied simplified and efficient protocols for differentiation of human iPSCs into defined populations of atrial or ventricular CM subtypes based on monolayer and feeder-free culture conditions. In particular, we combined different molecular and functional approaches like RNA sequencing, SILAC-based proteomics, optical AP and calcium imaging, and EHM analyses to deeply characterize the iPSC-aCMs and iPSC-vCMs. Our data demonstrate that iPSC-aCMs and -vCMs differ significantly in their gene and protein expression as well as in their functional properties. A comprehensive understanding of the molecular and functional characteristics of in vitro-generated iPSC-CM subtypes may provide possibilities to use these cells for a better understanding of cardiac subtype-restricted disease mechanisms and the development of heart chamber-specific pharmacological interventions (32).

While several monolayer-based protocols for differentiation of human iPSCs into ventricular CMs have been established $(11,13)$, protocols for atrial CM differentiation are currently less efficient $(9,14)$ or based on embryoid body formation in suspension culture $(10,14,15)$ or Matrigel sandwiches (16). Here, we applied a stage-specific activation of RA signaling to induce atrial CM differentiation in a monolayerbased and well-defined culture, which is less laborious and allows a simple implementation. By applying this protocol, we could show that iPSCs were efficiently differentiated into $90 \% \mathrm{cTNT}^{+} \mathrm{CMs}_{\text {, with the }}$ majority being atrial subtype $\left(95 \% \mathrm{MLC}^{2} \mathrm{~V}^{-} / \mathrm{MLC}^{2} \mathrm{~A}^{+}\right.$cells). We demonstrate that this protocol can be applied robustly to 3 iPSC lines of different origin (bone marrow, fibroblasts, and peripheral blood), which were generated by different reprogramming methods (STEMCAA lentivirus, Sendai virus). Our data confirm the specific action of RA at $1 \mu \mathrm{M}$ within a defined time window (days 3-6) during differentiation for the induction of iPSC-aCMs by upregulation of the RA-related genes $H O X A 1$ and $R A R B(10,33)$. In vivo studies could convincingly show that RA-induced expression of the anterior HOX genes HOXA1, HOXA3, and $H O X B 1$ in cardiac progenitor cells define the posterior domain of the secondary heart field, which further contributes to the atria $(8,34)$. We found that CM subtype specification occurs very early during cardiac differentiation (at stages of mesodermal and cardiac progenitor induction), which is consistent with previous reports (10). Our data are in line with previous studies showing that the modulation of WNT signaling resulted in the generation of $90 \% \mathrm{cTNT}^{+}{ }_{\mathrm{PPSC}-\mathrm{CMs}}$, with $89 \% \mathrm{MLC}^{2} \mathrm{~V}^{+} / \mathrm{MLC}^{2} \mathrm{~A}^{-}$or $\mathrm{MLC}_{2} \mathrm{~V}^{+} /$ $\mathrm{MLC}_{2} \mathrm{~A}^{+}$, indicating a ventricular subtype. Notably, so far none of the published protocols including ours could generate completely homogeneous populations of only one certain CM subtype.

Atrial and ventricular CMs exhibit distinct molecular and functional profiles essential for their diverse physiological roles in the heart. The molecular and functional characteristics of iPSC-aCMs and -vCMs were deeply investigated in this study to obtain a comprehensive understanding of the cardiac subtype identities. The global transcriptome and proteome profiles of the iPSC-vCMs and -aCMs obtained from RNA sequencing at day 90 and SILAC-based proteomics at day 68 overlap with the data from human fetal and adult ventricles and atria, respectively $(23,26,27,35-38)$. Consistent with previous data from human ventricular and atrial tissues $(23,35,36,38)$, we found generally accepted subtype-selective expression of genes encoding transcription factors, structural proteins, and ion channels crucial for ventricular or atrial CM development and function. Those typical for ventricular CMs, for example, HAND1, HEY2, and IRX4; MYL2, MYH7, and GJA1; as well as KCNJ2 and KCNJ4, respectively, were found in iPSC-vCMs, while those important for atrial CMs, for example, HEY1, TBX5, and NR2F2; MYL7, MYH6, and GJA5; as well as $K C N A 5, K C N J 3$, and $K C N K 3$, respectively, were found in iPSC-aCMs. In particular, our SILAC-based quantitative proteomics in mostly nondividing iPSC-CMs allowed identifying and quantitatively profiling more than 3,500 proteins with high accuracy, including those with lower expression levels.

Electrophysiological differences are considered major determinants within the CM subtypes caused by differentially expressed ion channels leading to subtype-specific AP morphologies (39). Strikingly, we were able to observe a subtype-specific expression pattern of the crucial ion channels $\mathrm{K}_{\mathrm{ir}} 2.1$ (KCNJ2) being predominantly expressed in iPSC-vCMs and $\mathrm{K}_{\mathrm{v}} 1.5$ (KCNA5), $\mathrm{K}_{\mathrm{ir}} 3.1$ (KCNJ3), and $\mathrm{K}_{2 \mathrm{p}} 3.1$ (KCNK3) being highly expressed in iPSC-aCMs. Mutations within the potassium channels $\mathrm{K}_{\mathrm{v}} 1.5, \mathrm{~K}_{\mathrm{ir}} 3.1$, and $\mathrm{K}_{2 \mathrm{p}} 3.1$ are often associated with subtype-selective cardiomyopathies like atrial fibrillation $(21,22,40)$. In accordance with the divergent ion channel occurrence, our functional analysis via optical AP and calcium imaging revealed that the differentially differentiated iPSC-aCMs and iPSC-vCMs display a typical subtype-specific shape and properties of APs and CaTs with comparable values of freshly isolated human aCMs and vCMs, respectively $(41,42)$. Compared with the generally used patch-clamp technique for electrophysiological measurements, noninvasive imaging-based methods with the voltage-sensitive dye di-8-ANEPPS and calcium dye Rhod-2 
AM, both with fast kinetics for accurate analysis, enable precise and parallel phenotyping of APs and CaTs in single CMs. Our data showed that the spontaneous beating frequency of iPSC-vCMs was significantly lower compared with iPSC-aCMs, which is in line with previously published data $(9,18)$. This beating rate difference might be explained by the differential subtype-specific expression of some ion channels. In our study, we found significantly higher expression of HCN1 (coding for pacemaker channel) and CACNA1H (coding for T-type calcium channel) and lower expression of KCNJ2 and KCNJ4 in iPSC-aCMs compared with iPSC-vCMs. Previous studies showed that an increase in the expression of the T-type calcium channel and HCN1 was associated with a dramatic increase in beating frequency of CMs $(43,44)$. The high expression of $K C N J 2$ and $K C N J 4$ is primarily responsible for maintenance of negative resting membrane potential and protects ventricular CMs from pacemaker activity (45). However, further studies are needed to clarify how the differential expression of the channel machinery might be responsible for the beating rate differences in atrial and ventricular CMs.

By generation of EHM from either iPSC-aCMs or iPSC-vCMs in a more native environment, in which the cells form intercellular connections and produce their own extracellular matrix, we aimed to recapitulate the in vivo physiology of the atrial and ventricular heart muscles. We were able to detect various similarities between iPSC-aEHM and iPSC-vEHM, such as morphological appearance or force of contraction, but we could also identify significant differences including beating frequency and chamber-specific pharmacological responses to $\beta$-adrenergic and muscarinergic stimulations $(29,31)$. Altogether, our data could corroborate that human atrial and ventricular myocardium can be recapitulated in IPSC-aEHM and -vEHM.

The availability of human CM subtypes and heart muscle models is increasingly considered to be important for a better understanding of the underlying molecular and functional mechanisms leading to cardiac diseases $(2,46,47)$. Various cardiac diseases are often restricted to a certain cardiac subtype. Atrial fibrillation, for example, is characterized by irregular atrial rhythm and decline in atrial mechanical function leading to an increased risk of heart failure and stroke (48). Subtype-specific iPSC-CMs - either in $2 \mathrm{D}$ or in complex multicellular atrial or ventricular EHM - also provide unprecedented access to human models in drug screening and cardiotoxicity approaches.

Despite the great potential of subtype-specific iPSC-CMs and -EHM over previous cardiac models, this technology still possesses certain limitations. A major concern is the immaturity of iPSC-CMs compared with freshly isolated adult CMs from the human heart. Adult CMs differ from iPSC-CMs with respect to shape, cell size, cellular structures, and metabolism by being far larger, rod-shaped, with large sarcomeric area, and possessing an extensive T-tubule network and large numbers of mitochondria (49). However, based on our findings and relevant published data, iPSC-CMs also recapitulate many important structural, molecular, and electrophysiological features, resembling CMs at embryonic or fetal stages (38). The iPSC-aCMs and iPSC-vCMs in long-term culture (days 60-100) display well-organized sarcomeric structures, functional calcium handling, and typical membrane ion channels that impact cardiac electrophysiology and function. Moreover, transcriptional and proteomic signatures of the iPSC-CMs as well as their functional characteristics like contractile force, electrical and calcium dynamics, and pharmacological responses are associated with functional maturation. The characteristics of maturity in iPSC-CMs depend strongly on time, as well as on cellular milieu and paracrine signaling. It is important to elucidate the underlying mechanisms of CM maturation and to develop methods and protocols to promote $\mathrm{CM}$ maturation under in vitro culture conditions. Recent studies reported that mechanical stimulation and electric pacing of EHM improved the structural, functional, and electrophysiological maturation of iPSC-CMs $(50,51)$. Additionally, biochemical cues like adrenergic stimulation or collaborative effects of thyroid hormone, IGF-1, and dexamethasone, as applied in our protocols for the generation of EHM, are expected to enhance the electrophysiology, bioenergetics, and contractile force generation of iPSC-CMs such that they resemble more mature phenotypes $(52,53)$. Despite these caveats, atrial and ventricular iPSC-CMs and -EHM are in our view highly attractive for future applications in disease modeling and for drug screening.

In summary, we applied an efficient and simple differentiation protocol to induce iPSCs to become a defined atrial CM subtype under a feeder-free and monolayer culture condition. This study for the first time to our knowledge combines different molecular and functional approaches like RNA sequencing, SILACbased proteomics, and tissue-based EHM analyses to deeply characterize the atrial and ventricular iPSCCMs. The comprehensive understanding of the molecular and functional characteristics of in vitro-generated iPSC-CM subtypes will improve their use in more precise disease modeling and chamber-specific drug development and might foster the development of novel and patient-tailored therapeutic strategies within the scope of personalized medicine to prevent and treat cardiovascular diseases. 


\section{Methods}

Generation and characterization of iPSCS. Human iPSC lines from 3 healthy donors were used in this study. Human iPSC lines iWT.D2.1 (GOEi001-A.1; FB2-iPS1) and iBM76.3 (GOEi005-A.3; MSC3-iPS3) were generated from dermal fibroblasts and mesenchymal stem cells, respectively, using the STEMCCA lentivirus system and were described previously (54). The human iPSC line isWT1.Bld2 (GOEi014-A.2) was generated from peripheral blood mononuclear cells (PBMCs) using the integration-free CytoTune-iPS 2.0 Sendai Reprogramming Kit (Thermo Fisher Scientific) with the reprogramming factors OCT4, KLF4, SOX2, and c-MYC. In brief, PBMCs were isolated from 10-ml donor blood samples and cultured in blood medium composed of 50\% IMDM with Glutamax (Thermo Fisher Scientific), 50\% Ham's F12-Nutrient Mix (Thermo Fisher Scientific), $5 \mathrm{mg} / \mathrm{ml}$ human recombinant albumin (Sigma-Aldrich), $1 \times$ chemically defined lipid concentrate (Thermo Fisher Scientific), $14 \mathrm{ng} / \mathrm{ml}$ sodium selenite (Sigma-Aldrich), $64 \mu \mathrm{g} /$ $\mathrm{ml}$ L-ascorbic acid 2-phosphate (Sigma-Aldrich), $10 \mu \mathrm{g} / \mathrm{ml}$ human recombinant insulin (Thermo Fisher Scientific), $100 \mu \mathrm{g} / \mathrm{ml}$ human recombinant transferrin (Sigma-Aldrich), $1 \mu \mathrm{M}$ dexamethasone (SigmaAldrich), $450 \mu \mathrm{M}$ 1-thioglycerol (Sigma-Aldrich), $2 \mathrm{U} / \mathrm{ml}$ human recombinant erythropoietin (R\&D Systems), $40 \mathrm{ng} / \mathrm{ml}$ human recombinant IGF-I (Peprotech), $10 \mathrm{ng} / \mathrm{ml}$ human recombinant IL-3 (Peprotech), $50 \mathrm{ng} / \mathrm{ml}$ human recombinant stem cell factor (Peprotech), $100 \mathrm{U} / \mathrm{ml}$ penicillin, and $100 \mu \mathrm{g} / \mathrm{ml}$ streptomycin (Thermo Fisher Scientific). After cultivation for 6-8 days, $2 \times 10^{5}$ PBMCs were plated in 1 well of a Geltrex-coated (Thermo Fisher Scientific) 12-well plate in blood medium and transduced with Sendai virus cocktail hKOS: hc-Myc: hKlf4 at a MOI of 5:5:3. Virus was removed after 24 hours and cells were then cultured in blood medium supplemented with $500 \mu \mathrm{M}$ sodium butyrate (Sigma-Aldrich). From day 7 after transduction, medium was changed to E8 medium (Thermo Fisher Scientific) supplemented with $500 \mu \mathrm{M}$ sodium butyrate (days 7-11) with daily medium change. Individual colonies with iPSC-like morphology typically appeared after 2-3 weeks and were transferred manually into Geltrex-coated 12-well plates in E8 medium supplemented with $2 \mu \mathrm{M}$ Thiazovivin (Merck Millipore). Established iPSC lines were maintained in Geltrex-coated plates, passaged with Versene solution (Thermo Fisher Scientific) and cultured in E8 medium supplemented with $2 \mu \mathrm{M}$ Thiazovivin on the first day after passaging for at least 10 passages before being used for pluripotency characterization and differentiation experiments.

Alkaline phosphatase activity in iPSC lines was detected using the Alkaline Phosphatase Kit (SigmaAldrich) according to manufacturer's instructions.

For karyotyping, iPSCs were arrested in metaphase using $10 \mathrm{ng} / \mathrm{ml}$ demecolcine (Sigma-Aldrich) at $37^{\circ} \mathrm{C}$ for 4 hours followed by resuspension of cells in total volume of $10 \mathrm{ml} 75 \mathrm{mM} \mathrm{KCl}$ at $37^{\circ} \mathrm{C}$ for 30 minutes. Cells were fixed 3 times with $4^{\circ} \mathrm{C}$ cold methanol/glacial acetic acid (3:1) for 10 minutes. Finally, cells were resuspended in $2 \mathrm{ml}$ methanol/glacial acetic acid and $20 \mu 1$ was dropped onto precooled objective slides, dried at room temperature (RT) overnight, and G-banded by standard Giemsa staining (SigmaAldrich). Karyotyping analysis was performed using the Axio Imager M2 microscopy system (Carl Zeiss) with CCD 1300 QDS camera (Visitron Systems) and Case Data Manager 6.0 (Applied Spectral Imaging).

Directed differentiation of iPSCs into CM subtypes. Directed differentiation of human iPSCs into iPSCvCMs was performed via WNT signaling modulation, as described with modifications $(11,13)$. In brief, differentiation was initiated at $80 \%-90 \%$ confluence in Geltrex-coated plates with cardio differentiation medium composed of RPMI 1640 with Glutamax and HEPES (Thermo Fisher Scientific), $0.5 \mathrm{mg} / \mathrm{ml}$ human recombinant albumin, and $0.2 \mathrm{mg} / \mathrm{ml} \mathrm{L}$-ascorbic acid 2-phosphate and sequential treatment with $4 \mu \mathrm{M}$ CHIR99021 (Merck Millipore) for 48 hours and then $5 \mu \mathrm{M}$ IWP2 (Merck Millipore) for 48 hours. Medium was changed to cardio culture medium composed of RPMI 1640 with Glutamax, HEPES, and 2\% B27 (Thermo Fisher Scientific) at day 8. For atrial subtype differentiation, $1 \mu \mathrm{M}$ RA (Sigma-Aldrich) was supplemented at days 3-6 during differentiation.

Differentiated cultures around day 15 were digested with collagenase B (Worthington Biochemical) and $0.25 \%$ Trypsin/EDTA (Thermo Fisher Scientific) and replated at lower density (1:2 to 1:3) in Geltrexcoated plates. Metabolic CM selection was performed using cardio selection medium composed of RPMI 1640 without glucose (Thermo Fisher Scientific), $0.5 \mathrm{mg} / \mathrm{ml}$ human recombinant albumin, $0.2 \mathrm{mg} / \mathrm{ml}$ L-ascorbic acid 2-phosphate, and $4 \mathrm{mM}$ lactate (Sigma-Aldrich) for 5 days. Afterwards, iPSC-CMs were cultured in cardio culture medium at least to day 60 for further maturation. Following differentiation, purity of iPSC-CMs was determined by flow cytometry analysis using an antibody against cTNT (a list of all antibodies used is provided in Supplemental Table 7). Differentiation efficiency was also determined by observation of contracting cells and only cultures covered with greater than $90 \%$ contracting areas were 
used for further experiments. Beating frequency of iPSC-CM cultures between days 60-97 was determined by counting spontaneous muscle contractions within 30 seconds of video recording. A minimum of 3 individual differentiation experiments of 3 iPSC lines were used for each analysis if not indicated otherwise.

Generation and analysis of EHM. Human iPSC lines iWT.D2.1 and isWT1.Bld2 were used for EHM experiments. Defined, serum-free EHM was generated from iPSC-CMs at day 60 and human foreskin fibroblasts (ATCC) at a 70:30 ratio, and contraction experiments were performed on 4-week-old EHM under isometric conditions, as previously described (51). Spontaneous beating frequency of EHM was assessed after a 10-minute equilibration phase. Afterwards, EHM was electrically stimulated at $2 \mathrm{~Hz}$ with 5 -ms square pulses of $200 \mathrm{~mA}$. EHM was mechanically stretched at intervals of $125 \mu \mathrm{m}$ until the maximum systolic force amplitude was observed according to the Frank-Starling law. Force-frequency response and response to drugs were assessed at individual $\mathrm{EC}_{50}$ calcium concentrations. Response to $10 \mu \mathrm{M}$ carbachol (Sigma-Aldrich) was tested in the presence or absence of prestimulation with $1 \mu \mathrm{M}$ isoprenaline (SigmaAldrich). Where indicated, spontaneous beating frequency changes after stimulation with carbachol was investigated without electrical stimulation. The CSA of EHM was measured as previously described (55). iPSC-CMs before EHM generation or after enzymatic dispersion of EHM were fixed in ice-cold 70\% ethanol and stained with anti- $\alpha$-actinin antibody as previously described (51).

Voltage and calcium imaging. For cytosolic calcium transient and voltage recordings, iPSC-CMs were replated at low density on Geltrex-coated coverslips and cultured for at least 7 days in cardio culture medium before measurement. For recordings, iPSC-CMs between days 94-141 were incubated with $5 \mu \mathrm{M}$ Rhod2 AM fluorescent calcium indicator (Thermo Fisher Scientific) and 0.02\% (w/v) Pluronic F-127 (Thermo Fisher Scientific) in Tyrode's solution containing $140 \mathrm{mM} \mathrm{NaCl}, 5.4 \mathrm{mM} \mathrm{KCl}, 1.8 \mathrm{mM} \mathrm{CaCl}_{2}, 1 \mathrm{mM}$ $\mathrm{MgCl}_{2}, 10 \mathrm{mM}$ HEPES, and $10 \mathrm{mM}$ glucose (pH 7.4) at RT for 20 minutes, followed by incubation with $10 \mu \mathrm{M}$ voltage-sensitive di-8-ANEPPS (Thermo Fisher Scientific) in Tyrode's solution at RT for another 10 minutes. Recordings obtained from paced cells were field stimulated at $0.5 \mathrm{~Hz}$ (18 V, $3 \mathrm{~ms}$ duration) and recorded in Tyrode's solution at RT with total medium change every 10 minutes. Images were captured in line-scan mode (512 pixels, $45 \mu \mathrm{m}, 1057.7 \mathrm{~Hz}, 20,000$ cycles, pinhole $6 \mathrm{AU}$ ) using the LSM 710 confocal microscopy system (Carl Zeiss) with a $63 \times / 1.4$ NA oil objective and Zen 2009 software. Rhod-2 AM was excited at $561 \mathrm{~nm}$, while emission was detected at 566-646 nm. Di-8-ANEPPS was excited at $458 \mathrm{~nm}$, while emission was detected at 530-580 nm (green channel; F1) and 582-700 nm (red channel; F2) and the ratio between the 2 different emission spectra was calculated for each line scan as previously described (56). Postacquisition analyses of changes in intracellular calcium and membrane potential were performed by plotting of mean signal intensity as a function of time using Image $(\mathrm{NIH})$, polynomial smoothing (6th order, 100 neighbors) of raw data using GraphPad Prism 7, and final analysis of APs and CaTs using the Peak Analysis Tool in LabChart Pro 8 (ADInstruments) with the following settings: automatic recognition of resting membrane potential, TStart $15 \%$ of height away from resting membrane potential, TRise and TFall is defined between $0 \%$ and $100 \%$ of the peak height. APD 20/50/80 and CaTD 20/50/80 were defined as the time from the maximum of the potential/transient until 20\%/50\%/80\% signal decay. Time to peak (TtP) of CaTs was defined as the time from start to the maximum of the transient. For each analyzed cell, the individual data set represents a mean of 5-9 AP/CaT recordings.

SILAC-based proteomics. The human iPSC line isWT1.Bld2 was used for SILAC experiments. Differentiated cultures were labeled with stable isotopes of arginine and lysine by long-term culture in SILAC cardio culture medium composed of SILAC RPMI 1640 (Thermo Fisher Scientific) with 2\% B27, $10 \mu 1 / \mathrm{ml}$ Glutamax, $25 \mathrm{mM}$ HEPES, $1.74 \mathrm{mM}$ L-proline (Sigma-Aldrich), and $0.219 \mathrm{mM}{ }^{13} \mathrm{C}_{6},{ }^{15} \mathrm{~N}_{2}$-L-lysine (Cambridge Isotopes) and $0.575 \mathrm{mM}^{13} \mathrm{C}_{6},{ }^{15} \mathrm{~N}_{4}$-L-arginine (Cambridge Isotopes) for heavy-isotope labeling or $0.219 \mathrm{mM}$ L-lysine (Sigma-Aldrich) and $0.575 \mathrm{mM}$ L-arginine (Sigma-Aldrich) for light-isotope labeling for up to 53 days (days 15-68), respectively. For cell lysis, cell pellets were incubated on ice for 30 minutes in $0.5 \%$ Nonidet P-40 buffer containing $50 \mathrm{mM}$ Tris/ $\mathrm{HCl}(\mathrm{pH} 7.8), 150 \mathrm{mM} \mathrm{NaCl}, 0.2 \%$-dodecyl $\beta$-Dmaltoside, $1 \mathrm{mM} \mathrm{Na}_{3} \mathrm{VO}_{4}, 5 \mathrm{mM} \mathrm{NaF}$, and complete protease inhibitors (Roche). Equal amounts of protein of light-labeled iPSC-vCMs were mixed with heavy-labeled iPSC-aCMs and vice versa. Mass spectrometric (MS) analyses were performed as described previously (57). Briefly, protein samples were separated by SDS-PAGE, digested in-gel with trypsin and analyzed by LC-MS/MS on a Q Exactive hybrid quadrupole/ orbitrap mass spectrometer (Thermo Fisher Scientific). Two technical replicates per sample were acquired. Raw data were processed using MaxQuant Software 1.5.2.8 (Max Planck Institute for Biochemistry, Martinsried). Proteins were identified against the UniProtKB Homo sapiens reference proteome (v2016.07; 
71,544 entries) augmented with common lab contaminants. The search was performed with trypsin as enzyme and iodoacetamide as cysteine-blocking agent. Up to 2 missed tryptic cleavages and methionine oxidation as a variable modification were allowed. Instrument type 'Orbitrap' was selected to adjust for MS acquisition specifics. The arginine R10 and lysine K8 labels including the 'Re-quantify' option were specified for relative protein quantification. Resulting data from MaxQuant analysis were further analyzed by Perseus (version 1.5.2.6, Max Planck Institute for Biochemistry) to obtain relative protein quantification values and to perform statistical evaluation. The data set was filtered based on a minimum of 2 valid values. Ratios of intensity of heavy- and light-labeled proteins were log2 transformed and the median of biological and technical replicates was calculated. Intensity values were $\log 10$ transformed. Next, outlier significance scores for log protein ratios depending on intensity values (significance B; see ref. 58) were calculated for each $\log 2$ ratio. To assess labeling efficiency, selected gel slices at different apparent molecular weights from SDS-PAGE separation of a non-mixed K8/R10-labeled sample were processed and analyzed as described above, and the percentage of heavy isotope-labeled peptides was calculated using an in-house R script.

Immunocytochemical staining and flow cytometry. For immunofluorescence studies, cells cultured on coverslips were fixed in 4\% paraformaldehyde (PFA; Carl Roth) at RT for 20 minutes and blocked with 1\% bovine serum albumin (BSA; Sigma Aldrich) in PBS at $4^{\circ} \mathrm{C}$ overnight. EHM was fixed in $4 \%$ PFA at RT for 1 hour, washed in PBS at $4^{\circ} \mathrm{C}$ overnight, immersed in $30 \%$ sucrose solution (w/v) at $4^{\circ} \mathrm{C}$ for 5 hours, and embedded in OCT embedding medium (Sakura Finetek) and horizontal cryosections were cut. For nuclear or sarcomere proteins, cells were permeabilized with 0.1\% Triton X-100 (Carl Roth) in 1\% BSA. Samples were mounted in Fluoromount-G (Thermo Fisher Scientific). Images were collected using the LSM 710 confocal microscopy system or using the Axio Observer Z1 microscopy system (Carl Zeiss).

For flow cytometry analysis of iPSC-CMs, the cells were dissociated with $0.25 \%$ Trypsin/EDTA into single cells, fixed with 4\% PFA at RT for 20 minutes, permeabilized with $0.1 \%$ Triton X-100, and blocked with $1 \% \mathrm{BSA}$ in $\mathrm{PBS}$ at $4^{\circ} \mathrm{C}$ for at least 2 hours. Cells were then incubated with the antibody against cTNT at $4^{\circ} \mathrm{C}$ overnight. After washing 3 times, cells were incubated with secondary antibody in $1 \%$ BSA in PBS at RT for 1 hour. Subsequently, cells were analyzed using the LSRII flow cytometer (BD Biosciences). Unstained and secondary-antibody-only stained cells were used as negative controls. At least 10,000 events were analyzed per sample.

Western blot. Pellets of iPSC-CMs were snap-frozen in liquid nitrogen and stored at $-80^{\circ} \mathrm{C}$. For cell lysis, cells were incubated on ice for 30 minutes in lysis buffer composed of $20 \mathrm{mM}$ Tris/ $\mathrm{HCl}(\mathrm{pH} 7.4)$, $200 \mathrm{mM} \mathrm{NaCl}, 1 \mathrm{mM} \mathrm{Na}_{3} \mathrm{VO}_{4}, 20 \mathrm{mM} \mathrm{NaF}, 1 \%$ Triton X-100, $1 \mathrm{mM}$ dithiothreitol, and PhosSTOP phosphatase inhibitor (Roche). After centrifugation at $13,000 \mathrm{~g}$ at $4^{\circ} \mathrm{C}$ for 5 minutes, protein concentration was determined using a bicinchoninic acid assay (Thermo Fisher Scientific) according to the manufacturer's instructions. Prior to Western blotting, samples were denatured at $37^{\circ} \mathrm{C}$ for 30 minutes. A total amount of $40 \mu \mathrm{g}$ protein was subsequently separated by sodium dodecyl sulfate-polyacrylamide gel electrophoresis (SDS-PAGE) using a $10 \%$ polyacrylamide gel with a $4.3 \%$ stacking gel at $25 \mathrm{mV}$ for approximately 60 minutes. Proteins were transferred to a nitrocellulose membrane at $400 \mathrm{~mA}$ on ice for 2 hours. Membranes were incubated with primary antibodies (Supplemental Table 7) diluted in either $2.5 \%$ milk or $1 \%$ BSA at $4^{\circ} \mathrm{C}$ overnight. After washing, secondary antibodies were applied at RT for 1 hour. To visualize the immunoreaction, an enhanced chemiluminescent detection kit (Thermo Fisher Scientific) was used.

Reverse transcriptase PCR and quantitative real-time PCR. Pellets of iPSCs or iPSC-CMs were snap-frozen in liquid nitrogen and stored at $-80^{\circ} \mathrm{C}$. Total RNA was isolated using the SV Total RNA Isolation System (Promega) according to the manufacturer's instructions. RNA (100 ng) was used for the first-strand cDNA synthesis by using MULV Reverse Transcriptase (Thermo Fisher Scientific) and Oligo d(T)16 (Thermo Fisher Scientific). For reverse transcriptase PCR, one-tenth of cDNA was used as PCR template and amplified using the GoTaq G2 DNA polymerase (Promega) according to the manufacturer's instructions. For semiquantitative analysis, the intensity of individual bands of gene expression was quantified using AlphaEaseFC 4.0 (Alpha Innotech) and values were normalized to GAPDH expression as housekeeping gene. For RA screening, the intensity of individual bands of cardiac marker gene expression was quantified and values were normalized to CM-restricted TNNT2 expression and to controls at day 30. Primer sequences, annealing temperatures, and cycles used for reverse transcriptase PCR analyses are listed in Supplemental Table 8.

Quantitative real-time PCR reactions were carried out using SYBR Green PCR master mix, MicroAmp Optical 384-well plates, and the 7900HT fast Real-time PCR system (all from Thermo Fisher Scientific) according to the manufacturer's instructions, with the following parameters: $95^{\circ} \mathrm{C}$ for 10 minutes, 
followed by 40 cycles at $95^{\circ} \mathrm{C}$ for 15 seconds and $60^{\circ} \mathrm{C}$ for 1 minute. After 40 cycles, melt curve analysis was performed to ensure specificity of the products. Negative controls, reference genes, and each cDNA sample were amplified independently on the same plate and in the same experimental run in triplicate. Analysis was conducted using the $\triangle \Delta C$ T method according to the SDS 2.4 program and values were normalized to GAPDH expression as housekeeping gene and to controls at day 30. Primer sequences for real-time PCR analyses are listed in Supplemental Table 8.

RNA sequencing. Total RNA of iPSC-CM cultures was isolated using the SV Total RNA Isolation System. RNA quality was assessed by measuring the RIN (RNA integrity number) using a Fragment Analyzer (Advanced Bioanalytical). Library preparation for RNA sequencing was performed using the TruSeq RNA Sample Prep Kit v2 (Illumina) starting from 200 ng of total RNA. Accurate quantification of cDNA libraries was performed by using the QuantiFluor dsDNA System (Promega). The size range of final cDNA libraries was determined by applying the DNA 1000 chip on the Fragment Analyzer (average 290-320 bp). cDNA libraries were amplified and sequenced by using the cBot and HiSeq2000 from Illumina (SR; $1 \times 50 \mathrm{bp} ; \sim 30-40$ million reads per sample). Sequence images were transformed with Illumina software BaseCaller to BCL files, which were demultiplexed to FastQ files with CASAVA v1.8.2. Quality check was done via FastQC (v0.10.0). Read alignment was performed using STAR aligner (v2.3.0e_r291) to the H. sapiens reference genome (assembly hg19) allowing for 2 mismatches within 50 bases. Subsequently, read counting was performed using featureCounts (v1.4.6-p1) and read counts were analyzed in the R/Bioconductor environment (v3.2.1, 2015-06-18) using the DESeq2 package (v1.8.1). Candidate genes were filtered using an absolute $\log 2$ fold change $>0.5$ and FDR-corrected $P$ value $<$ 0.05. Gene annotation was performed using $H$. sapiens entries via biomaRt $\mathrm{R}$ package (v2.24.0). Clustered gene ontology analysis regarding encoded protein function was performed based on annotations of the gene ontology database WEB-based GEne SeT AnaLysis Toolkit (http://webgestalt.org). The complete data set is available at the NCBI's Gene Expression Omnibus (GEO, http://www.ncbi.nlm. nih.gov/geo/) under GEO accession number GSE111007.

Statistics. Data are presented as the mean \pm standard error of the mean (SEM). Statistical analysis was performed with GraphPad Prism 7 using the D'Agostino-Pearson normality test, the nonparametric unpaired and 2-tailed Mann-Whitney $U$ test to compare differences between 2 independent groups or the 2-way ANOVA test with Sidak's correction for comparison of more groups and conditions. Results were considered statistically significant when the $P$ value was $<0.05\left({ }^{*} P<0.05\right.$; ${ }^{* *} P<0.01$; ${ }^{* *} P<0.001$; ${ }^{* * *} P<0.0001$; n.s., $\left.P>0.05\right)$.

Study approval. This study was approved by the Ethics Committee of the University Medical Center Göttingen (approval number: 21/1/11), and carried out in accordance with the approved guidelines. Samples from donors were collected under signed informed consent prior to the participation in the study.

\section{Author contributions}

LC and K. Guan designed the study. LC, MT, and K. Guan designed the experiments. LC, MT, KS, K. Gerstenberg, CL, SH, MS, and GS performed the experiments, acquired data, and analyzed the data. HB, WHZ, and GH gave technical support and conceptual advice. LC, MT, and K. Guan wrote and edited the manuscript.

\section{Acknowledgments}

We thank Y. Wedekind, L. Cyganek, M. Grohe, N. Gotzmann, Y. Hintz, and J. Appelhans for excellent technical assistance. B. Downie and O. Shomroni from the Transcriptome and Genome Analysis Laboratory (UMG) are acknowledged for their support. This work was supported by the DZHK (to L. Cyganek, K. Guan, and W.H. Zimmermann), by the DFG (GU 595/3-1 to K. Guan; SFB1002 A04, B04, C04, and S1 to K. Guan, M. Tiburcy, W.H. Zimmermann, and G. Hasenfuss; SFB937 TP18 to W.H. Zimmermann; IRTG1816 RP7 to K. Guan and RP12 to W.H. Zimmermann; and IPTG2251 to K. Guan), by the Bundesministerium für Bildung und Forschung (BMBF) grant e:Bio - Modul II - Verbundprojekt: CaRNAtion (031L0075C to K. Guan and G. Hasenfuss), by the Sächsische Aufbaubank grant PhänoKard to K. Guan, and by the Kinderherzstiftung (to H. Bohnenberger).

Address correspondence to: Kaomei Guan, Institute of Pharmacology and Toxicology, Technische Universität Dresden, Fetscherstraße 74, 01307 Dresden, Germany. Phone: 49.351.458.6246; Email: kaomei.guan@tu-dresden.de. 
1. Savla JJ, Nelson BC, Perry CN, Adler ED. Induced pluripotent stem cells for the study of cardiovascular disease. J Am Coll Cardiol. 2014;64(5):512-519.

2. Sayed N, Liu C, Wu JC. Translation of human-induced pluripotent stem cells: from clinical trial in a dish to precision medicine. J Am Coll Cardiol. 2016;67(18):2161-2176.

3. Cyganek L, Chen S, Borchert T, Guan K. Cardiac progenitor cells and their therapeutic application for cardiac repair. J Clin Exp Cardiolog. 2013;S11:008.

4. Kolanowski TJ, Antos CL, Guan K. Making human cardiomyocytes up to date: Derivation, maturation state and perspectives. Int J Cardiol. 2017;241:379-386.

5. Harvey RP. Patterning the vertebrate heart. Nat Rev Genet. 2002;3(7):544-556.

6. Vincent SD, Buckingham ME. How to make a heart: the origin and regulation of cardiac progenitor cells. Curr Top Dev Biol. 2010;90:1-41.

7. Ryckebusch L, et al. Retinoic acid deficiency alters second heart field formation. Proc Natl Acad Sci USA. 2008;105(8):2913-2918.

8. Stefanovic S, Zaffran S. Mechanisms of retinoic acid signaling during cardiogenesis. Mech Dev. 2017;143:9-19.

9. Zhang $Q$, et al. Direct differentiation of atrial and ventricular myocytes from human embryonic stem cells by alternating retinoid signals. Cell Res. 2011;21(4):579-587.

10. Lee JH, Protze SI, Laksman Z, Backx PH, Keller GM. Human pluripotent stem cell-derived atrial and ventricular cardiomyocytes develop from distinct mesoderm populations. Cell Stem Cell. 2017;21(2):179-194.e4.

11. Lian X, et al. Directed cardiomyocyte differentiation from human pluripotent stem cells by modulating $\mathrm{Wnt} / \beta$-catenin signaling under fully defined conditions. Nat Protoc. 2013;8(1):162-175.

12. Weng Z, et al. A simple, cost-effective but highly efficient system for deriving ventricular cardiomyocytes from human pluripotent stem cells. Stem Cells Dev. 2014;23(14):1704-1716.

13. Burridge PW, et al. Chemically defined generation of human cardiomyocytes. Nat Methods. 2014;11(8):855-860.

14. Devalla HD, et al. Atrial-like cardiomyocytes from human pluripotent stem cells are a robust preclinical model for assessing atrial-selective pharmacology. EMBO Mol Med. 2015;7(4):394-410.

15. Laksman Z, et al. Modeling atrial fibrillation using human embryonic stem cell-derived atrial tissue. Sci Rep. 2017;7(1):5268

16. Leyton-Mange JS, et al. Rapid cellular phenotyping of human pluripotent stem cell-derived cardiomyocytes using a genetically encoded fluorescent voltage sensor. Stem Cell Reports. 2014;2(2):163-170.

17. Chen Z, et al. Subtype-specific promoter-driven action potential imaging for precise disease modelling and drug testing in hiPSC-derived cardiomyocytes. Eur Heart J. 2017;38(4):292-301.

18. Pei F, et al. Chemical-defined and albumin-free generation of human atrial and ventricular myocytes from human pluripotent stem cells. Stem Cell Res. 2017;19:94-103.

19. Chuva de Sousa Lopes SM, et al. Patterning the heart, a template for human cardiomyocyte development. Dev Dyn. 2006;235(7):1994-2002.

20. Wang Z, Fermini B, Nattel S. Sustained depolarization-induced outward current in human atrial myocytes. Evidence for a novel delayed rectifier $\mathrm{K}^{+}$current similar to Kv1.5 cloned channel currents. Circ Res. 1993;73(6):1061-1076.

21. Dobrev $\mathrm{D}$, et al. The $\mathrm{G}$ protein-gated potassium current $\mathrm{I}(\mathrm{K}, \mathrm{ACh})$ is constitutively active in patients with chronic atrial fibrillation. Circulation. 2005;112(24):3697-3706.

22. Schmidt C, et al. Upregulation of $\mathrm{K}(2 \mathrm{P}) 3.1 \mathrm{~K}^{+}$current causes action potential shortening in patients with chronic atrial fibrillation. Circulation. 2015;132(2):82-92.

23. Asp J, Synnergren J, Jonsson M, Dellgren G, Jeppsson A. Comparison of human cardiac gene expression profiles in paired samples of right atrium and left ventricle collected in vivo. Physiol Genomics. 2012;44(1):89-98.

24. Mann M. Functional and quantitative proteomics using SILAC. Nat Rev Mol Cell Biol. 2006;7(12):952-958.

25. Ong SE, Foster LJ, Mann M. Mass spectrometric-based approaches in quantitative proteomics. Methods. 2003;29(2):124-130.

26. Lu ZQ, Sinha A, Sharma P, Kislinger T, Gramolini AO. Proteomic analysis of human fetal atria and ventricle. J Proteome Res. 2014;13(12):5869-5878.

27. Doll S, et al. Region and cell-type resolved quantitative proteomic map of the human heart. Nat Commun. 2017;8(1):1469.

28. Nerbonne JM, Kass RS. Molecular physiology of cardiac repolarization. Physiol Rev. 2005;85(4):1205-1253.

29. Mulieri LA, Hasenfuss G, Leavitt B, Allen PD, Alpert NR. Altered myocardial force-frequency relation in human heart failure. Circulation. 1992;85(5):1743-1750.

30. Sossalla S, et al. Altered $\mathrm{Na}(+)$ currents in atrial fibrillation effects of ranolazine on arrhythmias and contractility in human atrial myocardium. J Am Coll Cardiol. 2010;55(21):2330-2342.

31. Du XY, Schoemaker RG, Bos E, Saxena PR. Characterization of the positive and negative inotropic effects of acetylcholine in the human myocardium. Eur J Pharmacol. 1995;284(1-2):119-127.

32. Eschenhagen T, Mummery C, Knollmann BC. Modelling sarcomeric cardiomyopathies in the dish: from human heart samples to iPSC cardiomyocytes. Cardiovasc Res. 2015;105(4):424-438.

33. Simandi Z, Balint BL, Poliska S, Ruhl R, Nagy L. Activation of retinoic acid receptor signaling coordinates lineage commitment of spontaneously differentiating mouse embryonic stem cells in embryoid bodies. FEBS Lett. 2010;584(14):3123-3130.

34. Bertrand N, et al. Hox genes define distinct progenitor sub-domains within the second heart field. Dev Biol. 2011;353(2):266-274.

35. Ellinghaus $\mathrm{P}$, et al. Comparing the global mRNA expression profile of human atrial and ventricular myocardium with highdensity oligonucleotide arrays. J Thorac Cardiovasc Surg. 2005;129(6):1383-1390.

36. Barth AS, et al. Functional profiling of human atrial and ventricular gene expression. Pflugers Arch. 2005;450(4):201-208.

37. Piccini I, Rao J, Seebohm G, Greber B. Human pluripotent stem cell-derived cardiomyocytes: Genome-wide expression profiling of long-term in vitro maturation in comparison to human heart tissue. Genom Data. 2015;4:69-72.

38. van den Berg CW, et al. Transcriptome of human foetal heart compared with cardiomyocytes from pluripotent stem cells. Development. 2015;142(18):3231-3238.

39. Voigt N, Dobrev D. Atrial-selective potassium channel blockers. Card Electrophysiol Clin. 2016;8(2):411-421

40. Christophersen IE, et al. Genetic variation in KCNA5: impact on the atrial-specific potassium current IKur in patients with lone 
atrial fibrillation. Eur Heart J. 2013;34(20):1517-1525.

41. Amos GJ, Wettwer E, Metzger F, Li Q, Himmel HM, Ravens U. Differences between outward currents of human atrial and subepicardial ventricular myocytes. J Physiol (Lond). 1996;491(Pt 1):31-50.

42. Voigt N, et al. Cellular and molecular mechanisms of atrial arrhythmogenesis in patients with paroxysmal atrial fibrillation. Circulation. 2014;129(2):145-156.

43. Lalevée N, et al. Aldosterone increases T-type calcium channel expression and in vitro beating frequency in neonatal rat cardiomyocytes. Cardiovasc Res. 2005;67(2):216-224.

44. Le Menuet D, Munier M, Meduri G, Viengchareun S, Lombès M. Mineralocorticoid receptor overexpression in embryonic stem cell-derived cardiomyocytes increases their beating frequency. Cardiovasc Res. 2010;87(3):467-475.

45. Grant AO. Cardiac ion channels. Circ Arrhythm Electrophysiol. 2009;2(2):185-194.

46. Gintant G, Sager PT, Stockbridge N. Evolution of strategies to improve preclinical cardiac safety testing. Nat Rev Drug Discov. 2016;15(7):457-471

47. Passier R, Orlova V, Mummery C. Complex tissue and disease modeling using hiPSCs. Cell Stem Cell. 2016;18(3):309-321.

48. Zoni-Berisso M, Lercari F, Carazza T, Domenicucci S. Epidemiology of atrial fibrillation: European perspective. Clin Epidemiol. 2014;6:213-220

49. Yang X, Pabon L, Murry CE. Engineering adolescence: maturation of human pluripotent stem cell-derived cardiomyocytes. Circ Res. 2014;114(3):511-523

50. Ruan JL, et al. Mechanical stress conditioning and electrical stimulation promote contractility and force maturation of induced pluripotent stem cell-derived human cardiac tissue. Circulation. 2016;134(20):1557-1567.

51. Tiburcy M, et al. Defined engineered human myocardium with advanced maturation for applications in heart failure modeling and repair. Circulation. 2017;135(19):1832-1847.

52. Yang X, et al. Tri-iodo-l-thyronine promotes the maturation of human cardiomyocytes-derived from induced pluripotent stem cells. J Mol Cell Cardiol. 2014;72:296-304.

53. Birket MJ, et al. Contractile defect caused by mutation in MYBPC3 revealed under conditions optimized for human PSC cardiomyocyte function. Cell Rep. 2015;13(4):733-745.

54. Streckfuss-Bömeke K, et al. Comparative study of human-induced pluripotent stem cells derived from bone marrow cells, hair keratinocytes, and skin fibroblasts. Eur Heart J. 2013;34(33):2618-2629.

55. Streckfuss-Bömeke K, et al. Severe DCM phenotype of patient harboring RBM20 mutation S635A can be modeled by patientspecific induced pluripotent stem cell-derived cardiomyocytes. J Mol Cell Cardiol. 2017;113:9-21.

56. Tian Q, et al. Optical action potential screening on adult ventricular myocytes as an alternative QT-screen. Cell Physiol Biochem. $2011 ; 27(3-4): 281-290$.

57. Atanassov I, Urlaub H. Increased proteome coverage by combining PAGE and peptide isoelectric focusing: comparative study of gel-based separation approaches. Proteomics. 2013;13(20):2947-2955.

58. Cox J, Mann M. MaxQuant enables high peptide identification rates, individualized p.p.b.-range mass accuracies and proteomewide protein quantification. Nat Biotechnol. 2008;26(12):1367-1372. 